\title{
Reconstruction of two-dimensional magnetopause structures from Cluster observations: verification of method
}

\author{
H. Hasegawa ${ }^{1}$, B. U. Ö Sonnerup ${ }^{1}$, M. W. Dunlop ${ }^{2}$, A. Balogh ${ }^{3}$, S. E. Haaland ${ }^{4,5}$, B. Klecker ${ }^{5}$, G. Paschmann ${ }^{4,5}$, \\ B. Lavraud ${ }^{6}$, I. Dandouras ${ }^{6}$, and H. Rème ${ }^{6}$ \\ ${ }^{1}$ Thayer School of Engineering, Dartmouth College, Hanover, NH, USA \\ ${ }^{2}$ Space Sciences Division, Space Science and Technology Department, Rutherford Appleton Laboratory, Chilton, UK \\ ${ }^{3}$ Space and Atmospheric Physics Group, Imperial College, London, UK \\ ${ }^{4}$ International Space Science Institute, Bern, Switzerland \\ ${ }^{5}$ Max-Planck-Institut für extraterrestrische Physik, Garching, Germany \\ ${ }^{6}$ Centre d'Etude Spatiale des Rayonnements, Toulouse, France
}

Received: 23 April 2003 - Revised: 28 August 2003 - Accepted: 4 September 2003 - Published: 2 April 2004

\begin{abstract}
A recently developed technique for reconstructing approximately two-dimensional $(\partial / \partial z \approx 0)$, timestationary magnetic field structures in space is applied to two magnetopause traversals on the dawnside flank by the four Cluster spacecraft, when the spacecraft separation was about $2000 \mathrm{~km}$. The method consists of solving the GradShafranov equation for magnetohydrostatic structures, using plasma and magnetic field data measured along a single spacecraft trajectory as spatial initial values. We assess the usefulness of this single-spacecraft-based technique by comparing the magnetic field maps produced from one spacecraft with the field vectors that other spacecraft actually observed. For an optimally selected invariant $(z)$-axis, the correlation between the field components predicted from the reconstructed map and the corresponding measured components reaches more than 0.97 . This result indicates that the reconstruction technique predicts conditions at the other spacecraft locations quite well.

The optimal invariant axis is relatively close to the intermediate variance direction, computed from minimum variance analysis of the measured magnetic field, and is generally well determined with respect to rotations about the maximum variance direction but less well with respect to rotations about the minimum variance direction. In one of the events, field maps recovered individually for two of the spacecraft, which crossed the magnetopause with an interval of a few tens of seconds, show substantial differences in configuration. By comparing these field maps, time evolution of the magnetopause structures, such as the formation of magnetic islands, motion of the structures, and thickening of the magnetopause current layer, is discussed.
\end{abstract}

Key words. Magnetospheric physics (Magnetopause, cusp, and boundary layers) - Space plasma physics (Experimental and mathematical techniques, Magnetic reconnection)

Correspondence to: $\mathrm{H}$. Hasegawa

(hasegawa@dartmouth.edu)

\section{Introduction}

The magnetopause current layer has long been a focus of investigation, because physical processes operating in this region control energy and mass transfer from the solar wind into the magnetosphere. In most past studies, the structure of this boundary was examined under the assumption that it is locally one-dimensional (1-D), having spatial variations only in the direction parallel to $\boldsymbol{n}$, the vector normal to the boundary surface. The determination of $\boldsymbol{n}$ has usually been based on the assumption that the magnetopause is totally planar and has a fixed orientation during a traversal. These studies paid special attention to the normal components of plasma flow and field, because they are directly related to net transport of mass and energy across the magnetopause and to dynamic behavior. However, in reality, the magnetopause layer could have significant two- or three-dimensionality and/or temporal variations. If this is the case, previous analyses might in some cases have been misleading.

A technique utilizing single-spacecraft data to recover two-dimensional (2-D) magnetic structures in space has recently been developed and applied to magnetopause traversals (Sonnerup and Guo, 1996; Hau and Sonnerup, 1999; Hu and Sonnerup, 2000, 2003) and to flux rope observations in the solar wind (Hu and Sonnerup, 2001, 2002; Hu et al., 2003). In a proper frame of reference (the deHoffmannTeller frame), where the structures are assumed to be magnetohydrostatic, time-stationary, and have invariance along the $z$ direction, the equation $\boldsymbol{j} \times \boldsymbol{B}=\nabla p$ holds and can be reduced to the Grad-Shafranov (GS) equation in the $(x, y, z)$ Cartesian coordinate system (e.g. Sturrock, 1994):

$\frac{\partial^{2} A}{\partial x^{2}}+\frac{\partial^{2} A}{\partial y^{2}}=-\mu_{0} \frac{d P_{t}}{d A}=-\mu_{0} j_{z}(A)$,

where the partial magnetic vector potential, $A(x, y) \hat{z}$, is defined such that $\boldsymbol{B}=\left(\partial A / \partial y,-\partial A / \partial x, B_{z}(A)\right)$. The transverse pressure, $P_{t}=\left(p+B_{z}^{2} / 2 \mu_{0}\right)$, the sum of plasma pressure and pressure from the axial magnetic field, and hence, 
the axial current density $j_{z}$, are functions of $A$ alone. The plane GS Eq. (1) is solved numerically as a Cauchy problem using plasma and magnetic field measurements along a spacecraft trajectory through the structures as spatial initial values. As a result, the magnetic field configuration and plasma pressure distribution are obtained in a region of the $x-y$ plane surrounding the trajectory.

This data analysis technique has been fully developed and described in detail by Hau and Sonnerup (1999), and successfully tested by use of synthetic data from several analytic solutions (Hau and Sonnerup, 1999; Hu and Sonnerup, 2001, 2003; Hu et al., 2003). Because the method is based on the magnetohydrostatic equation, in which inertia forces are neglected, its application to magnetopause traversals is, strictly speaking, limited to cases in which reconnection effects are weak or absent. This means that the local structure can be approximately regarded as a tangential discontinuity (TD). Note, however, that our definition of TD includes not only the traditional 1-D current sheet with no normal magnetic field component $\left(B_{n}=0\right)$, but also 2-D or 3-D current layers having structured field lines within a TD. The presence of internal structures, such as magnetic islands and localized channels of magnetic flux linking the two sides of the magnetopause, is allowed, unless the inertia terms contribute significantly to the momentum balance. In the simplest application, a constant deHoffmann-Teller (HT) frame velocity, $\boldsymbol{V}_{H T}$, which describes the motion of the magnetic field structure past the spacecraft, is determined from standard HT analysis, using measured magnetic field vectors and plasma flow velocities (e.g. Khrabrov and Sonnerup, 1998). Then a co-moving frame, where the spacecraft moves across the structure with the velocity, $-\boldsymbol{V}_{H T}$, is defined such that $\hat{\boldsymbol{x}}=-\frac{\boldsymbol{V}_{H T}-\left(\boldsymbol{V}_{H T} \cdot \hat{z}\right) \hat{z}}{\left|\boldsymbol{V}_{H T}-\left(\boldsymbol{V}_{H T} \cdot \hat{z}\right) \hat{z}\right|}$ and $\hat{\boldsymbol{y}}=\hat{z} \times \hat{\boldsymbol{x}}$. The magnetic potential, $A$, at points along the $x$-axis, i.e. along the projection of the spacecraft trajectory onto the $x-y$ plane, is calculated by integrating the measured $B_{y}$ component of the field:

$A(x, 0)=\int_{0}^{x} \frac{\partial A}{\partial \xi} d \xi=-\int_{0}^{x} B_{y}(\xi, 0) d \xi$.

The space increment along the $x$-axis is obtained from the corresponding time increment via the constant HT frame velocity: $d \xi=-\boldsymbol{V}_{H T} \cdot \hat{\boldsymbol{x}} d t$. Since, as a result of the invariance in the $z$ direction, the quantities, $p(x, 0)$ and $B_{z}(x, 0)$ are both known along the $x$-axis, a functional fit of $P_{t}(x, 0)$ versus $A(x, 0)$ is used to approximate the function $P_{t}(A)$ on the right-hand side of the GS Eq. (1). Once $\frac{d P_{t}}{d A}$ is known along the trajectory, it can be used in the entire domain in the $x-$ $y$ plane that is threaded by field lines (given by $A=$ const.) encountered by the spacecraft. Outside of that domain, simple extrapolation of $P_{t}(A)$ is used. The integration proceeds explicitly in the $\pm y$ direction, starting at $y=0$ and utilizing $B_{x}(x, 0)=\left.\frac{\partial A}{\partial y}\right|_{y=0}, B_{y}(x, 0)=-\left.\frac{\partial A}{\partial x}\right|_{y=0}$, and $A(x, 0)$ as initial values (Hau and Sonnerup, 1999). As a result, the magnetic potential, $A(x, y)$, is obtained in a rectangular domain surrounding the $x$-axis. The contour plot of $A(x, y)$, called a field map or transect, represents the transverse magnetic field lines. The field component $B_{z}(x, y)$ and the plasma pressure $p(x, y)$ are computed from functions $B_{z}(A)$ and $p(A)$, obtained by fitting to the measurements along the spacecraft trajectory.

Determination of the orientation of the invariant $(z)$-axis is an important issue. If the spacecraft trajectory intersects a field line more than once, which commonly happens in magnetic flux rope observations, one can usually find the correct $z$-axis from single-spacecraft data by use of the condition that the three quantities, $p, B_{z}$, and $P_{t}$, take the same values at each intersection point (Hu and Sonnerup, 2002). For magnetopause traversals, however, multiple encounters of the same field line occur only near the center of the current sheet, whereas many other field lines are encountered only once. Furthermore, field lines encountered on the magnetospheric and magnetosheath sides of the boundary have pairwise the same $A$ value but usually have different $P_{t}$ values, indicating that the function $P_{t}(A)$ has two branches ( $\mathrm{Hu}$ and Sonnerup, 2003). This kind of behavior makes reliable determination of the invariant $(z)$-axis difficult: one can use only very few data points within the central current sheet for optimization of the choice of invariant axis and the resulting data fit to the functions $P_{t}(A), p(A)$, and $B_{z}(A)$. Because of this difficulty, the intermediate variance direction, computed from minimum variance analysis of the measured magnetic field (e.g. Sonnerup and Scheible, 1998), was often used as a proxy for the invariant axis in earlier studies (Hau and Sonnerup, 1999; Hu and Sonnerup, 2003).

In the present study, the reconstruction technique, as improved by Hu and Sonnerup (2003), is applied to two magnetopause traversals by the Cluster spacecraft, both occurring in the tail flank on the dawn side. In a previous study, using data from the AMPTE/IRM and UKS spacecraft, the spacecraft separation distance was only about $40 \mathrm{~km}$ and the resulting two field maps showed only minor differences $(\mathrm{Hu}$ and Sonnerup, 2000). For the events addressed in this paper, the four spacecraft formed a tetrahedron and were separated by about two thousand $\mathrm{km}$ from each other, allowing us to evaluate the model assumptions, such as two-dimensionality and time independence, and also to determine the orientation of an approximate invariant ( $z$ )-axis with more accuracy. In Sect. 2, we test the reconstruction technique with a Cluster event in which the encountered magnetopause appears as an approximately time-stationary current layer of the TD-type. In Sect. 3, we apply the method, as an experiment, to an event showing non-negligible temporal variations for which the reconstruction results obtained separately for two of the spacecraft are quite different. In the last section, we summarize our results and discuss their significance and implications. Our procedure to select an optimal invariant axis is described in Appendix A. 


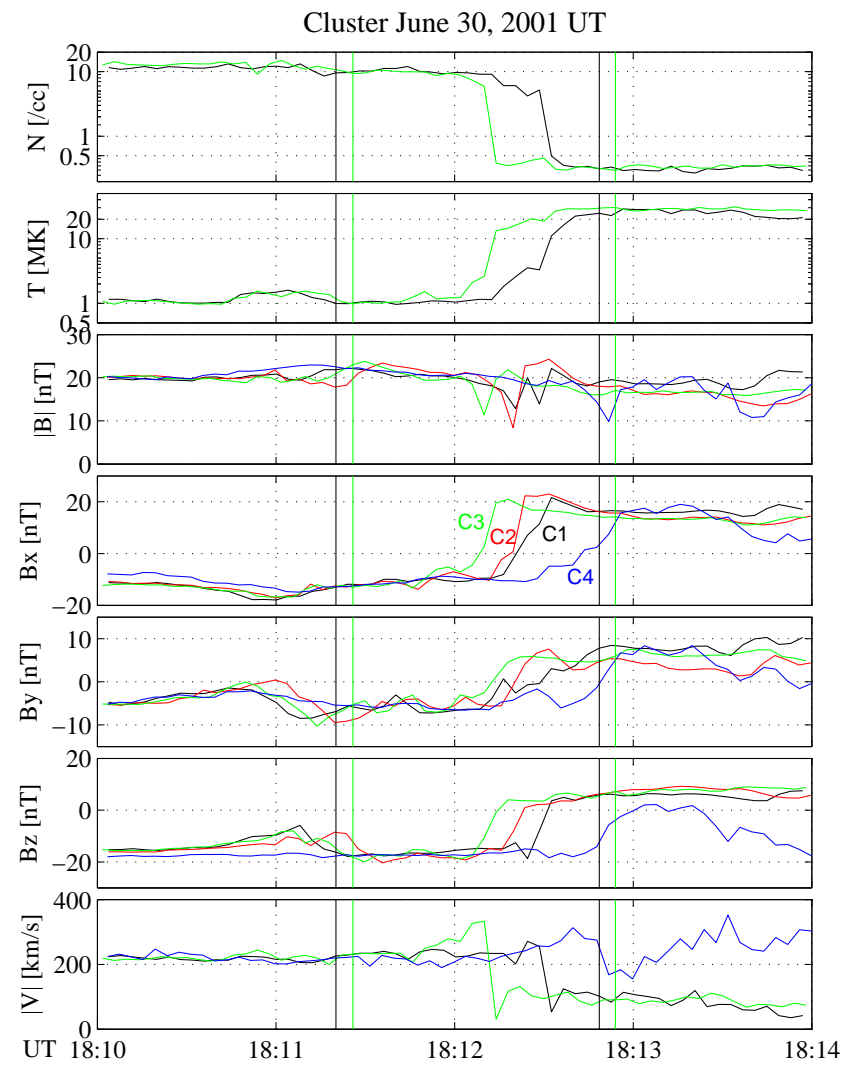

Fig. 1. Time series of Cluster measurements around a magnetopause crossing event occurring at $(-7.89,-17.11,3.25) R_{E}$ in GSE on 30 June 2001. The panels, from top to bottom, show ion number density, ion temperature, intensity and three components of the magnetic field in GSE coordinates, and ion bulk speed, respectively (black: spacecraft 1 (C1), red: C2, green: C3, blue: C4). The interval enclosed by the two black vertical lines is used in the reconstruction based on $\mathrm{C} 1$ data, while that enclosed by the green lines is in the reconstruction based on $\mathrm{C} 3$ data.

\section{Cluster event on 30 June 2001, 18:12 UT}

\subsection{Background information}

We utilize data from the Cluster Ion Spectrometry (CIS) and the Flux Gate Magnetometer (FGM) instruments. The CIS instruments measure full 3-D ion distribution functions and moments (Rème et al., 2001), with a resolution up to the spin rate $(\sim 4 \mathrm{~s})$. The FGM experiment can provide magnetic field measurements at time resolutions up to 120 vector samples/s (Balogh et al., 2001), but only spin-averaged data with $\sim 4$-s time resolution are used throughout this study. For our two events, the CIS instruments were fully operational on spacecraft 1 and 3 (C1 and C3). Additionally, after appropriate recalibration, the CODIF portion of CIS on board C4 delivered reliable velocity measurements. The FGM instruments on all four spacecraft were operating for the two events. However, since the reconstruction requires reliable pressure measurements, field maps can be produced only from $\mathrm{C} 1$ and $\mathrm{C} 3$.

On 30 June 2001, around 18:12 UT, the Cluster spacecraft were moving from northern high-latitude regions toward the

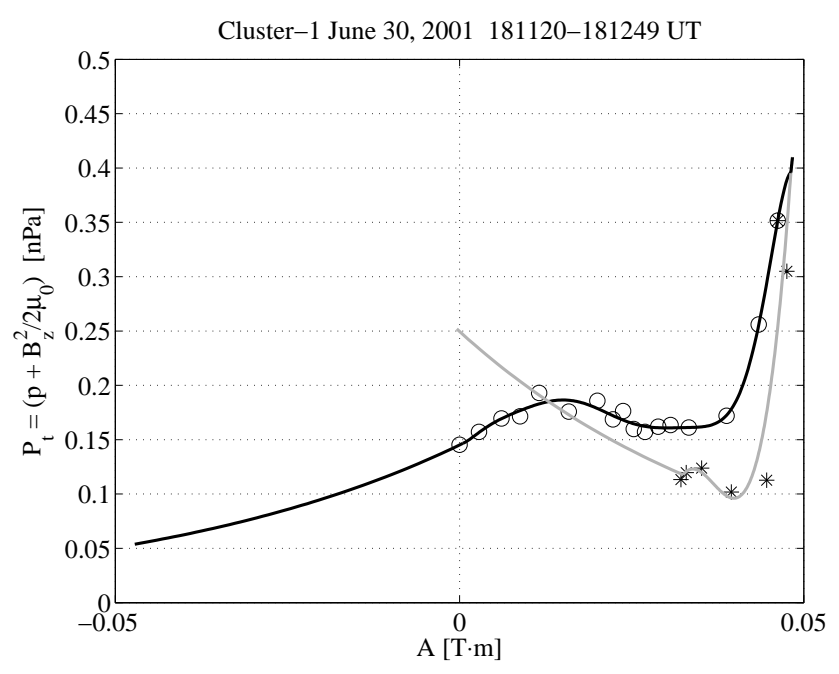

Fig. 2. Plot of transverse pressure $P_{t}$ versus computed vector potential $A$ and the fitting curves for the $\mathrm{C} 1$ magnetopause crossing on 30 June 2001. The circles and stars are data used in producing the magnetosheath (black curve) and magnetospheric (gray curve) branches, respectively. Extrapolated parts for each branch are shown; these are outside the measurements but are required for the reconstruction.

tail flank on the dawn side. An inbound, complete crossing of the magnetopause occurred when the reference spacecraft (C3) was located at $(X, Y, Z) \sim(-7.89,-17.11,3.25) R_{E}$ in the GSE coordinate system. Shown in Fig. 1 are, from top to bottom, time plots of ion number density, ion temperature, magnitude and three components of the magnetic field in GSE, and ion bulk speed, respectively. The black, red, green, and blue lines represent the measurements by $\mathrm{C} 1, \mathrm{C} 2$, $\mathrm{C} 3$, and $\mathrm{C} 4$, respectively. Plasma data for $\mathrm{C} 1$ and $\mathrm{C} 3$ are provided by the CIS/HIA instrument, which detects ions without mass discrimination. The velocity data for $\mathrm{H}^{+}$ions are provided by the CIS/CODIF instrument on board $\mathrm{C} 4$. The figure shows that the Cluster spacecraft were in the magnetosheath, which is characterized by high density $\left(\mathrm{N} \sim 10 \mathrm{~cm}^{-3}\right)$ and low temperature $(\mathrm{T} \sim 1 \mathrm{MK})$, until $\sim 18: 12 \mathrm{UT}$, although signatures associated with a flux transfer event (FTE), such as magnetic field perturbations and a temperature enhancement (for a review, see Elphic, 1995), were found at around 18:11 UT. The local magnetosheath magnetic field was tailward/dawnward/southward. The spacecraft then crossed the magnetopause and entered the plasma sheet where the temperature is much higher $(\sim 20 \mathrm{MK})$ and, in this event, the field magnitude is slightly smaller than in the magnetosheath. The orientation changes of the magnetic field indicate that the time order of the magnetopause traversals was $\mathrm{C} 3, \mathrm{C} 2, \mathrm{C} 1$, and $\mathrm{C} 4$.

We used the following criteria when selecting this crossing as a good test case: (1) The slope of the regression line in the Walén plot (e.g. Khrabrov and Sonnerup, 1998) is small, indicating strongly subalfvénic flow in the HT frame. This means that the boundary encountered is likely to be TDlike rather than RD-like, and that inertia effects associated 


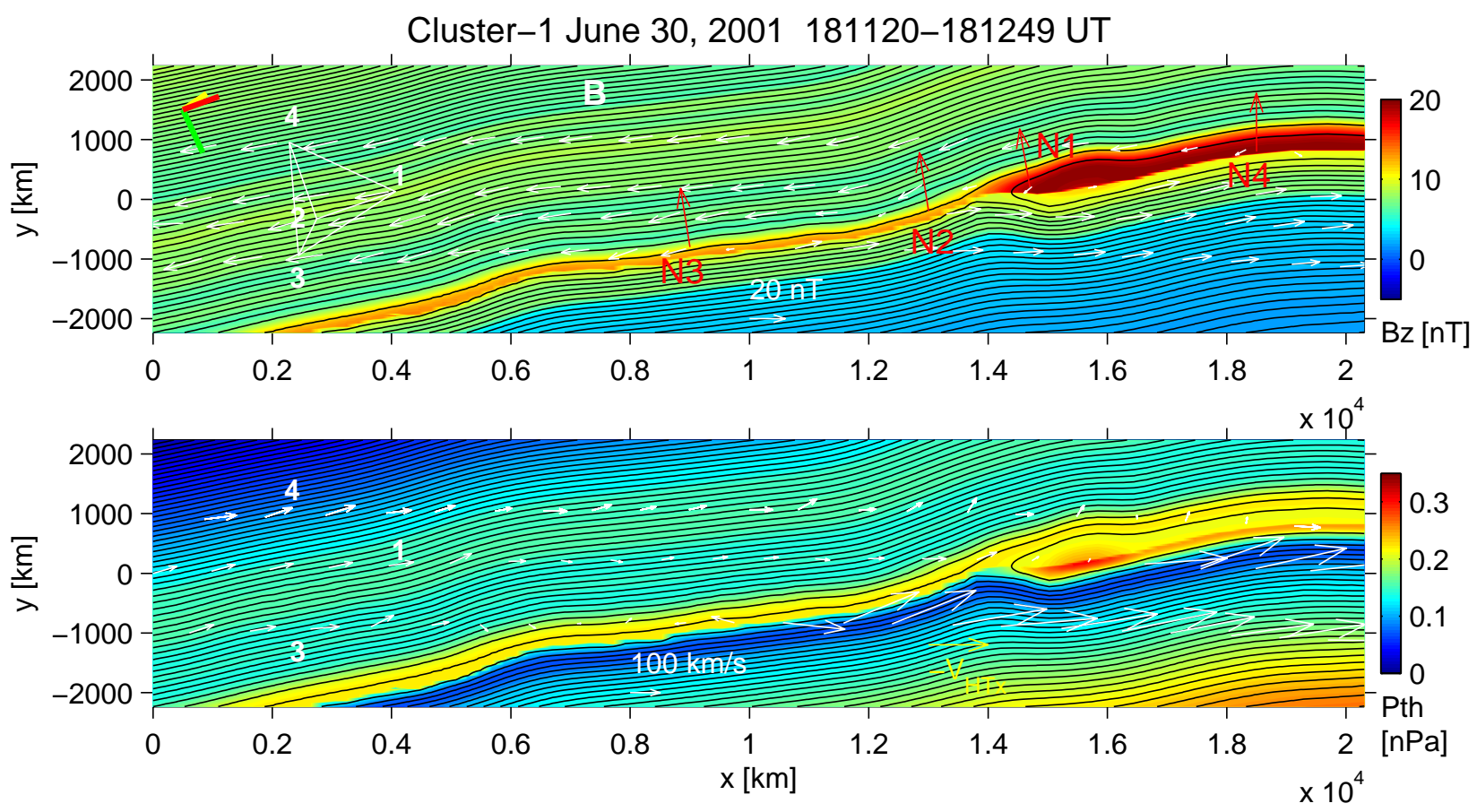

Fig. 3. Magnetic transect (top) and plasma pressure distribution (bottom) obtained by using time-varying HT frame velocity for the C1 magnetopause crossing on 30 June 2001. Contours describe the transverse magnetic field lines. In this reconstruction plane, the spacecraft generally moved from left to right: The magnetosheath, where $B_{x}<0 ; B_{y}<0$, is on the upper left side and the magnetosphere $\left(B_{x}>0 ; B_{y}>0\right)$ on the lower right side. In the top panel, $B_{z}$ is expressed in color as indicated by the color bar; the four spacecraft tetrahedron configuration is shown by white lines; the measured magnetic field vectors are projected as white arrows along the spacecraft trajectories; the normal vectors, N1 - N4, computed from MVABC, are projected as red arrows. Line segments in the upper left corner are projections of GSE unit vectors, $X$ (red), $Y$ (green), and $Z$ (yellow), onto the $x-y$ plane. In the bottom panel, the plasma pressure is shown in color; the ion bulk velocity vectors from CIS/HIA (C1 and C3) or CIS/CODIF (C4), transformed into the accelerating HT frame, are projected as white arrows.

with field-aligned plasma flows can be neglected. (2) A good deHoffmann-Teller frame with a constant HT velocity is found. This indicates that motion and time evolution of the structures are negligibly small in the HT frame and also that the MHD frozen-in condition is well satisfied. (3) The speed of the boundary motion along $\boldsymbol{n}$, calculated, for example, as $\boldsymbol{V}_{H T} \cdot \boldsymbol{n}$, is small enough to give a sufficient number of measurements within the magnetopause current layer, so as to allow for a good functional fitting of $P_{t}(A)$ and accurate recovery of meso-scale current sheet structures. These criteria can be used for identifying events for which the model assumptions are likely to hold and which are therefore suitable for the reconstruction analysis.

The time interval between the two black vertical lines is used for reconstruction from $\mathrm{C} 1$ data, whereas that between the green lines in the figure is for reconstruction from $\mathrm{C} 3$. These intervals include a number of data samples in both the magnetosheath and in the magnetosphere. Their start times are chosen such that variations related to the FTE are outside the intervals. The reason for this choice is that temporal variations and/or inertia effects, which cannot be taken into account in the current technique, could be significant in the FTE structures. We assume in this study that only ions, assumed to be protons with isotropic temperature, $T=\left(2 T_{\perp}+T_{\|}\right) / 3$, contribute to the plasma pressure.

\subsection{Reconstruction from spacecraft 1 crossing}

For the magnetopause encountered by $\mathrm{C} 1$, the Walén slope (slope of the regression line in a scatter plot of the velocity components in the HT frame, $\boldsymbol{V}-\boldsymbol{V}_{H T}$, versus the corresponding components of the Alfvén velocity, $\boldsymbol{B} / \sqrt{\mu_{0} \rho}$ ) is 0.3430 . The slope is much smaller than unity, indicating small field-aligned velocities in the HT frame, a result that is consistent with a TD. The minimum variance analysis (e.g. Sonnerup and Scheible, 1998) of the magnetic fields, measured by $\mathrm{C} 1$ in the interval 18:12:00-18:12:49 UT and using the constraint $\left\langle B_{n}\right\rangle=0$, (referred to as MVABC, hereinafter) yields the magnetopause normal vector, $\boldsymbol{n}=(0.2003$, $-0.9654,0.1671)$ in GSE. For this crossing and throughout this paper, we use the variance analysis with this constraint, because, for all the crossings examined in this study, the analysis without the constraint results in a rather small ratio of the intermediate to minimum eigenvalues $(<5)$ so that the normal determined may not be reliable. The usage of the constraint is justified for this event, since the Walén test shows consistency with a TD. The HT analysis for the same interval results in a constant HT frame velocity, $\boldsymbol{V}_{H T}=(-236.6,-83.9,-8.5) \mathrm{km} / \mathrm{s}$, with the correlation coefficient between the components of $-\boldsymbol{V} \times \boldsymbol{B}$ from the set of discrete measurements and the corresponding component of 


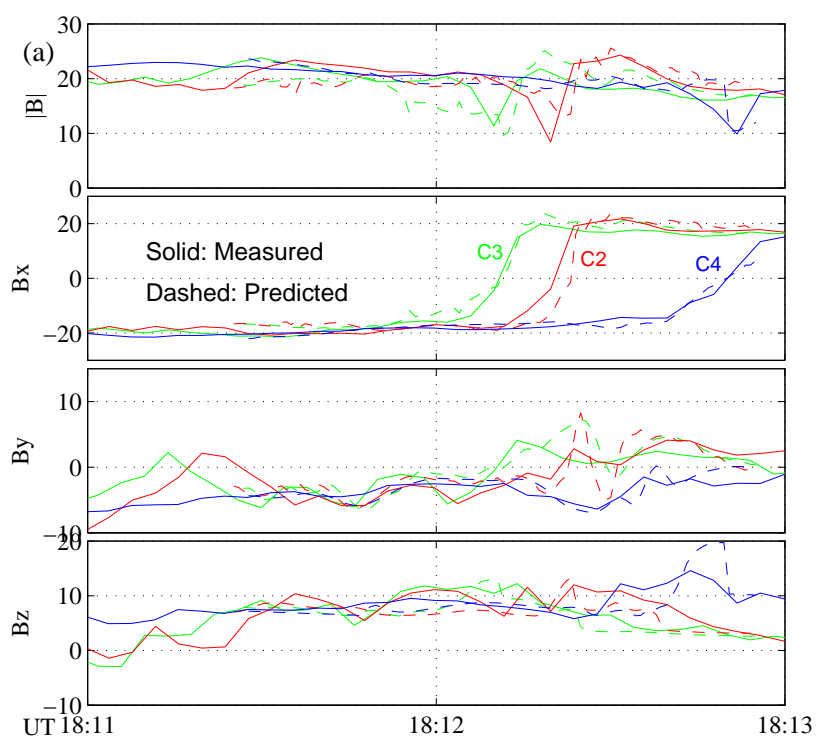

Correlation between Measured and Predicted B (B map from SC1)

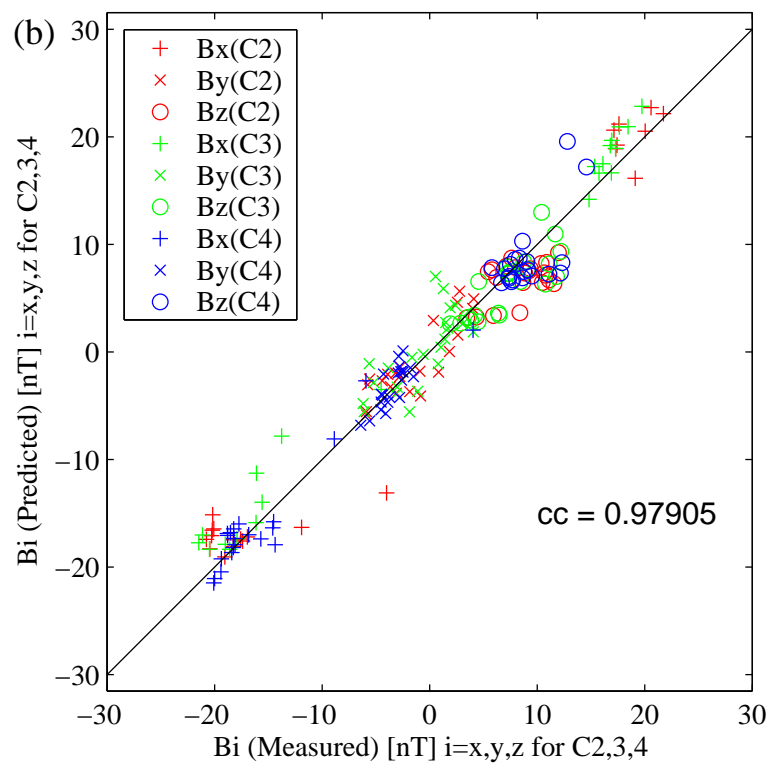

Fig. 4. (a) Time series of intensity and three components of the measured (solid) and predicted (dashed) magnetic field data (nT) in the reconstruction coordinates. The predicted data are based on the field map recovered from the $\mathrm{C} 1$ data (Fig. 3). (b) Correlation between the measured and predicted magnetic field components. The $x, y$, and $z$ components in the reconstruction coordinates are represented as a plus, a cross, and a circle, respectively. The curves and points are color-coded as in Fig. 1.

$-\boldsymbol{V}_{H T} \times \boldsymbol{B}$ being $c c_{H T}=0.9753$, indicating that a relatively good deHoffmann-Teller frame was found for this boundary (the value $c c_{H T}=1$ corresponds to an ideal HT frame). The magnetopause motion along $\boldsymbol{n}$ is $\boldsymbol{V}_{H T} \cdot \boldsymbol{n}=+32.2 \mathrm{~km} / \mathrm{s}$. The positive sign indicates outward magnetopause motion as expected for an inbound crossing.

By following the procedure described in Appendix A, the orientation of the optimal invariant axis was found to be $\hat{z}=(0.5941,-0.1160,-0.7960)$ in GSE. Figure 2 shows the resulting data points of $P_{t}$ versus $A$, obtained from the spacecraft measurements and the corresponding fitting curves for two separate branches. In constructing the diagram, we have used a slightly modified reconstruction technique in which the use of a sliding-window HT calculation is incorporated so as to allow for temporal variations in the velocity of the magnetopause structures as they move past the spacecraft ( $\mathrm{Hu}$ and Sonnerup, 2003); the reason for this procedure will be mentioned later. The sliding-window HT analysis yields a set of HT frame velocities, $\left\{\tilde{\boldsymbol{V}}_{H T}\right\}$, one vector for each data point sampled during the analysis interval. The $\tilde{V}_{H T}$ vectors vary from point to point along the spacecraft trajectory, resulting in a curved spacecraft trajectory in the reconstruction $(x-y)$ plane. The calculation of the magnetic potential $A$ is then modified to a line integral along the curved trajectory,

$$
\begin{aligned}
A= & \int \frac{\partial A}{\partial x} d x+\int \frac{\partial A}{\partial y} d y \\
& =\int-B_{y}\left\{-\tilde{\boldsymbol{V}}_{H T}\right\} \cdot \hat{\boldsymbol{x}} d t+\int B_{x}\left\{-\tilde{\boldsymbol{V}}_{H T}\right\} \cdot \hat{\boldsymbol{y}} d t .
\end{aligned}
$$

The black curve in Fig. 2 is the magnetosheath branch of $P_{t}(A)$, fitted by a high-order polynomial to the data samples (open circles) in the magnetosheath and in the central current sheet, a region of intense axial current density (large slope of $P_{t}(A)$ ). The gray curve, fitted to the data samples (stars) obtained on the magnetospheric side, is the magnetosphere branch of $P_{t}(A)$. Exponential functions, attached beyond the measured $A$ range, are used to generate the field map in regions of the $x-y$ plane containing field lines that are not encountered along the trajectory. We describe a reasonable way to determine the extrapolating functions in Appendix A.

The recovered magnetopause transect and the plasma pressure distribution are shown in Fig. 3. An improved numerical scheme, developed by $\mathrm{Hu}$ and Sonnerup (2003), was used to suppress numerical instabilities and hence, to extend the computation domain in the $y$ direction. The spatial extent in the $x$ direction corresponds to the analysis interval marked in Fig. 1. The spacecraft were moving to the right, as shown in the upper reconstruction map. C2, C3, and C4 were located away from $\mathrm{C} 1$ by $-1468 \mathrm{~km},+369 \mathrm{~km}$, and $+27 \mathrm{~km}$, respectively, in the out-of-plane $(z)$ direction. The contours show the transverse magnetic field lines, $\boldsymbol{B}_{t}=B_{x} \hat{\boldsymbol{x}}+B_{y} \hat{\boldsymbol{y}}$, separated by equal flux; color filled contours show the $B_{z}$ (upper panel) and $p$ (lower panel) distributions, as specified by the color bars. The white arrows along the spacecraft trajectories in the upper panel show the measured magnetic field vectors, projected onto the $x-y$ plane. The recovered field lines are exactly parallel to these vectors at $\mathrm{C} 1$ and also approximately parallel at the locations of the other spacecraft $(\mathrm{C} 2, \mathrm{C} 3$, and C4). The magnetosheath is located on the upper left side, where $B_{x}<0$ and $B_{y}<0$, while the magnetosphere is on the lower right side, where $B_{x}>0$ and $B_{y}>0$. The magnetopause encountered is found to be a thin, markedly nonplanar current layer of the TD-type. The presence of an $X$ point is evident at $(x, y) \approx(13500.0) \mathrm{km}$, resulting in a small number 


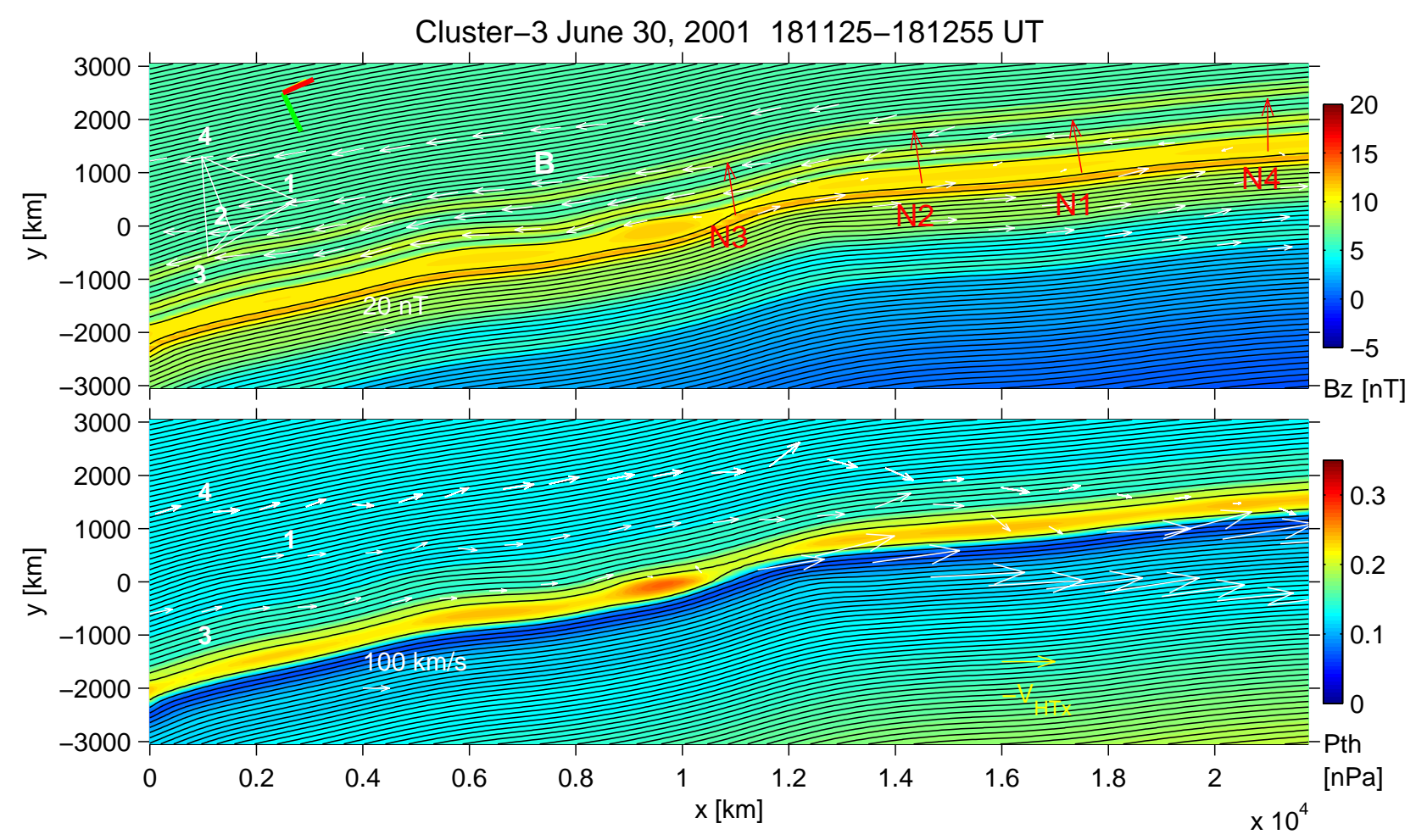

Fig. 5. Magnetic transect (top) and plasma pressure distribution (bottom) recovered by using a time-varying HT frame velocity for the C3 magnetopause crossing on 30 June 2001. The format and the spatial scale size are the same as in Fig. 3.

of interconnected field lines, embedded in the TD, and presumably in a localized thickening of the current sheet on the right of the $X$ point. The red arrows show the normal vectors computed from MVABC, based on each spacecraft measurement, and projected onto the $x-y$ plane. These normal directions are qualitatively consistent with the overall orientation of the recovered magnetopause surface but individual normals can deviate substantially from the local orientation (for example, see the normal for $\mathrm{C} 2$ ). The lower panel shows that the plasma pressure had a maximum in the central current layer. The white arrows in this panel represent the projection of the flow velocity vectors, as seen in the time-dependent HT frame. With a few exceptions, the vectors are approximately field-aligned for all three spacecraft, as they ideally should be. The velocities in the co-moving frame are not very large on the magnetosheath side, whereas they have substantial values on the magnetosphere side. Thus the HT frame moves approximately with the magnetosheath flow. However, the larger speeds in the magnetosphere contribute little to inertia forces because the corresponding streamlines, which ideally would coincide with the field lines, have no significant curvature.

Figure 4a shows the comparison between the time series of measured magnitude and the three components along the reconstruction coordinates of the magnetic field, and the corresponding values computed from the map recovered from $\mathrm{C} 1$. The predicted values were obtained along the trajectories of $\mathrm{C} 2, \mathrm{C} 3$, and $\mathrm{C} 4$ in Fig. 3. The time scale of the panel is expanded, relative to Fig. 1, to cover only the reconstructed range. We see that the recovered variations agree qualitatively with the measured variations for almost the whole interval. The recovered values predict the timings of the magnetopause crossings at the other spacecraft rather well, although the durations of the current layer traversals have small differences. Figure $4 \mathrm{~b}$ illustrates that a very good correlation exists between the measured and predicted magnetic field values for $\mathrm{C} 2, \mathrm{C} 3$, and $\mathrm{C} 4$ : the correlation coefficient is $c c=0.979$. This result indicates that the reconstruction technique based on $\mathrm{C} 1$ data is rather successful in predicting quantitatively reasonable values at the locations of the other three spacecraft.

The magnitude of this correlation coefficient can be used as a measure for judging whether or not the orientation of the invariant $z$-axis, the co-moving (HT) frame velocity, and the extrapolating exponential functions in the $P_{t}$ versus $A$ plot, are adequately selected. In fact, the optimal invariant axis, the HT frame, and the functional form $P_{t}(A)$ for the extrapolated parts are all determined in such a way that the correlation between measured and predicted magnetic field data is at, or near, a maximum. The steps we have used for optimal selection of the invariant axis, the HT frame, and the extrapolating functions are presented in Appendix A.

For the reconstruction in Fig. 3, the results were found to improve by use of the sliding-window HT method, suggesting that the whole set of magnetopause structures was approximately time-independent but was moving with small 


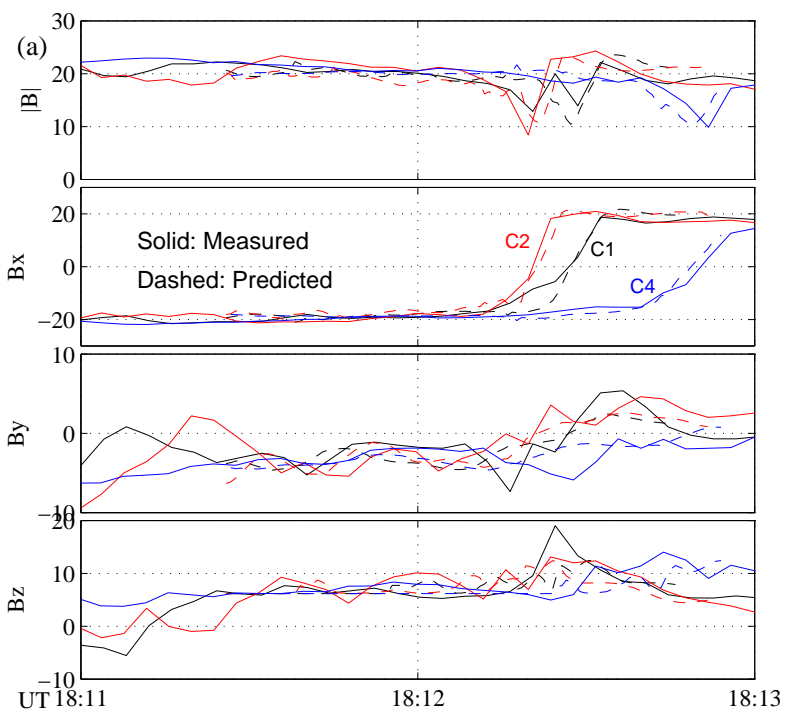

Correlation between Measured and Predicted B (B map from SC3)

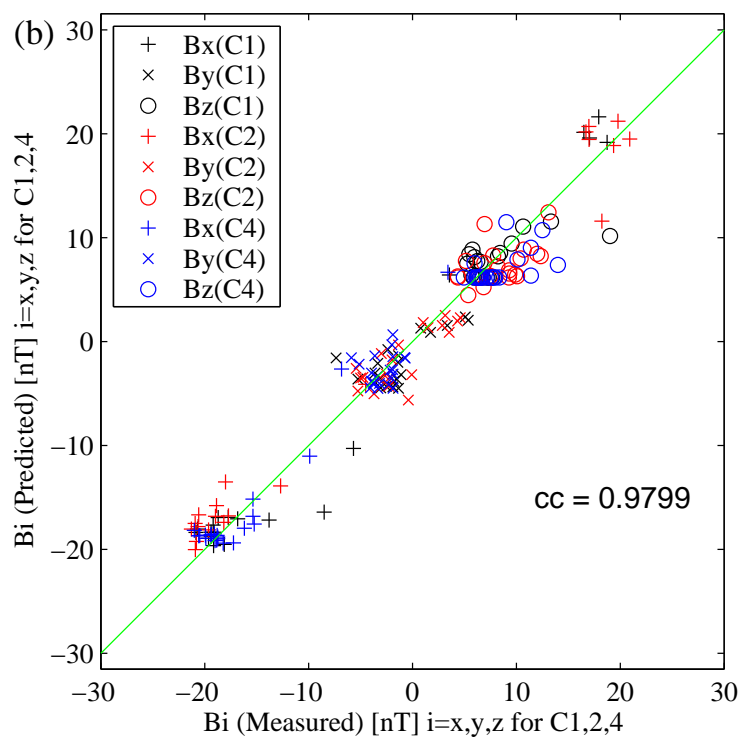

Fig. 6. (a) Time series of the measured (solid) and predicted (dashed) magnetic field data. The predicted data are based on the field map reconstructed from C3 data (Fig. 5). (b) Correlation between the measured and predicted magnetic field data. The format is the same as in Fig. 4.

acceleration. The extended reconstruction technique, developed by Hu and Sonnerup (2003), was shown to be useful for this case. The orientation of the selected invariant axis ( $z$-axis in Fig. 3) corresponds to the angles (defined in Appendix A) $\theta=-1^{\circ}$ and $\phi=6^{\circ}$, i.e. it was rotated away from the intermediate variance direction by $\sim 6^{\circ}$, with the axis of rotation mainly being the maximum variance direction (see Appendix B for a method to determine the intermediate and maximum variance directions under the constraint $\left\langle B_{n}\right\rangle=0$ ).

\subsection{Reconstruction from spacecraft 3 crossing}

The reconstruction technique is now applied to the magnetopause traversal by $\mathrm{C} 3$ which crossed the boundary $\sim 20 \mathrm{~s}$ earlier than $\mathrm{C} 1 \mathrm{did}$, using the data interval denoted by two
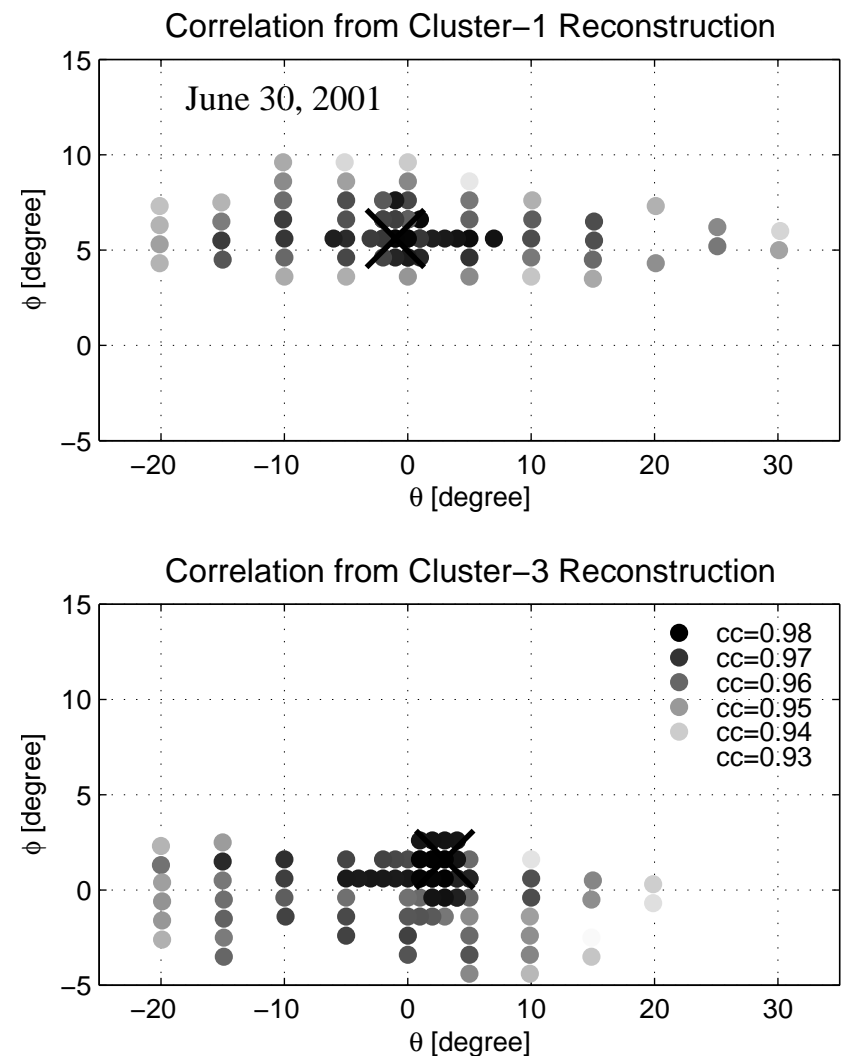

Fig. 7. Dependence of the correlation coefficient between the measured and predicted field data on the choice of the invariant $(z)$-axis for the reconstructions based on the $\mathrm{C} 1$ (upper panel) and $\mathrm{C} 3$ (lower panel) data; see text for definition of the angles $\theta$ and $\phi .(\theta, \phi)=(0$, 0 ) corresponds to the intermediate variance direction determined by MVABC for the $\mathrm{C} 1$ magnetopause crossing. The orientations of the invariant axes used in producing Figs. 3 and 5 are shown as thick crosses. Note that the vertical and horizontal scales are different.

vertical green lines in Fig. 1. The MVABC and HT analysis yield: $\boldsymbol{n}=(0.2117,-0.9608,0.1791)$; a constant HT velocity, $\boldsymbol{V}_{H T}=(-269.4,-98.3,-14.8) \mathrm{km} / \mathrm{s}$, from the interval 18:11:41-18:12:38 UT. The correlation coefficient is $c c_{H T}=0.9598$, and $\boldsymbol{V}_{H T} \cdot \boldsymbol{n}=+34.7 \mathrm{~km} / \mathrm{s}$. The Walén slope is 0.3689 , indicating again that inertia effects due to fieldaligned flow were reasonably small. As before, these results are consistent with the spacecraft crossing an outward moving magnetopause of the TD-type.

For this case, the reconstruction, using neither the standard (constant HT frame speed) nor the sliding-window HT analysis, led to a satisfactory correlation between the predicted and measured magnetic field components. These results, and also the fact that the HT frame was less well determined $\left(c c_{H T}=0.9598\right)$, suggest that the motion of the structures varied rapidly and by significant amounts along the C3 trajectory. Therefore, before the reconstruction was performed, we modified the $y$ component (in the reconstruction plane) of the HT velocity vectors computed from the slidingwindow HT method, such that the remaining velocity vectors became completely parallel to the local magnetic field 
measured along the spacecraft $(\mathrm{C} 3)$ trajectory in the reconstruction plane. The obtained $P_{t}(A)$ profile (not shown) is qualitatively similar to that for the $\mathrm{C} 1$ reconstruction: $P_{t}(A)$ has two branches and has smaller values at smaller $A$, but it generally increases with $A$ and the two branches merge in the largest $A$ range. The magnetic field and pressure maps thus recovered are shown in Fig. 5. The trajectories of the spacecraft are more strongly bent than in Fig. 3, because of the substantial modification of the $y$ component of $\boldsymbol{V}_{H T}$ needed at certain points. By definition, the alignment between the flow vectors and the transverse field lines is now fulfilled for C3. The invariant axis is found to be $z=(0.6261$, $-0.0246,-0.7794)$ in GSE which is obtained by rotating the intermediate variance $(M)$ axis, computed from the $\mathrm{C} 1$ data, by $\theta=3^{\circ}$ and $\phi=2^{\circ}$. Thus, this orientation has an angle of $5.6^{\circ}$ with respect to the invariant axis used in Fig. 3, indicating that the two axes are not far away from one another. As in the previous case (Fig. 3), a qualitative agreement of the normal vectors from MVABC with the orientation of the recovered magnetopause is seen in Fig. 5. Interestingly, the global shape of the magnetopause surface is similar in the two maps - the one from $\mathrm{C} 1$ (Fig. 3) and the one from $\mathrm{C} 3$ (Fig. 5). The $X$ point, seen in Fig. 3 at $x=13500 \mathrm{~km}$, seems to be equivalent to the one found at $(x, y) \approx(11500.0) \mathrm{km}$ in Fig. 5, although its location in $y$ is displaced: it is between the $\mathrm{C} 1$ and $\mathrm{C} 2$ trajectories in Fig. 3, and between $\mathrm{C} 2$ and $\mathrm{C} 3$ in Fig. 5. If our interpretation is correct, the migration distance of the $X$ point of about $2000 \mathrm{~km}$ during the $\sim 20$-s time interval between the $\mathrm{C} 3$ and $\mathrm{C} 1$ crossings gives a sunward speed of the $X$ point of about $100 \mathrm{~km} / \mathrm{s}$ in the reconstruction plane. However, that plane was moving downtail at speed $\boldsymbol{V}_{H T} \cdot \hat{\boldsymbol{x}} \approx 230 \mathrm{~km} / \mathrm{s}$. Therefore, relative to Earth, the $X$ point was sliding tailward at some $130 \mathrm{~km} / \mathrm{s}$. The presence of the bulge in the magnetopause seen in Fig. 3 but absent in Fig. 5, may indicate a minor time evolution: it may have been produced as a result of ongoing reconnection activity at the $X$ point. The current layer thickness appears to be somewhat different. A small magnetic island located at $(x, y) \approx(9500.0) \mathrm{km}$ in Fig. 5 , where both the $B_{z}$ and the plasma pressure reach maximum values, is not found in Fig. 3. These differences in fine structures are due to the fact that the profile of the function $P_{t}(A)$ was quantitatively different, in and near the current sheet, for $\mathrm{C} 1$ and $\mathrm{C} 3$ (not shown), i.e. it may have been different on opposite sides of the dominant $X$ point. The structures in the current layer on the left side of the $X$ point in Fig. 5, where the field lines were not encountered by $\mathrm{C} 1$, thus may not have been recovered correctly in Fig. 3.

The time series of the measured magnetic field magnitude and components and the corresponding predicted values shown in Fig. 6a indicate that the reconstruction results predict both the timings and durations of the current layer crossing very well. In Fig. 5, C1, C2, and C4 were separated from C3 by $-325 \mathrm{~km},-1848 \mathrm{~km}$, and $-480 \mathrm{~km}$, respectively, in the $z$ direction. It is noteworthy that the predicted and measured variations are quite similar even for $\mathrm{C} 2$, whose $z$ position was farthest from $\mathrm{C} 3$, supporting the conclusion that the approximate invariance along the selected invariant $(z)$ axis held over the spatial scale of at least $2000 \mathrm{~km}$. Figure $6 \mathrm{~b}$ shows that an excellent correlation $(c c=0.980)$ between the measured and predicted field components is attained for this case, demonstrating that the technique succeeds in predicting the conditions in regions surrounding the spacecraft trajectory with reasonable accuracy.

\subsection{Orientation of invariant axis}

Figure 7 shows the dependence of the correlation between the measured and predicted field components on the choice of the invariant $(z)$-axis. $\theta$ and $\phi$ are the angles described in Appendix A: $(\theta, \phi)=(-90,0),(0,0)$, and $(0,90)$ correspond to the maximum, intermediate, and minimum variance directions, respectively, for the $\mathrm{C} 1$ magnetopause crossing. The intermediate variance direction computed from the $\mathrm{C} 3$ data is oriented toward $(\theta, \phi) \approx(2,-1)$. The correlation coefficient is shown by the darkness of the grey points. The thick cross represents the orientation of the optimal invariant axis used in Figs. 3 and 5. The spaces in the diagram where no points are shown correspond to axis orientations for which an unrealistic field map is recovered, either due to an unreasonable profile in the $P_{t}$ versus $A$ plot, or to the correlation coefficient being smaller than 0.93 . The optimal invariant axis is found to be relatively close to the intermediate variance axis, for both the $\mathrm{C} 1$ and the $\mathrm{C} 3$ reconstructions. The correlation coefficient is sensitive to changes in $\phi$ (rotation about the maximum variance direction) but less sensitive to changes in $\theta$ (rotation about the minimum variance direction), for both cases. In other words, the magnetic field configuration in the reconstructed map is strongly modified by changes in $\phi$ while it is only weakly sensitive to changes in $\theta$, the latter result being the finding also reported by Hau and Sonnerup (1999) and Hu and Sonnerup (2003).

\subsection{Summary of 30 June 2001 event}

Intercomparison of the two reconstructed maps (Figs. 3 and 5) demonstrates that the magnetopause encountered in this event was a quasi-static, TD-type current layer, for which the model assumptions appear to be well justified. Similarities of the orientation of the invariant axis, current sheet thickness, and the overall magnetopause structures among the results from $\mathrm{C} 1$ and $\mathrm{C} 3$ data indicate that mainly two-dimensional structures were present, with superimposed weak threedimensionality and temporal variations. The dominant $X$ point in the two maps appears to be a real feature, moving tailward, relative to Earth, at about $130 \mathrm{~km} / \mathrm{s}$. The associated magnetic topology allows for easy access of the magnetosheath plasma to the inner portion of the magnetopause layer, by means of field-aligned flow on the two sides of the $X$. 


\section{Cluster event on 5 July 2001, 06:23 UT}

\subsection{Background information}

The second event is a crossing from the magnetosphere to the magnetosheath occurring on 5 July 2001, around 06:23 UT, when $\mathrm{C} 3$ was located at $\sim(-6.78,-14.97,6.24) R_{E}$ in GSE. This event has also been investigated in detail by Haaland et al. (2004), with the objective of comparing singleand multi-spacecraft determinations of magnetopause orientation, speed, and thickness. Time plots of number density, temperature, magnitude and three GSE components of the magnetic field, and bulk flow speed are shown in Fig. 8. Compared to the 30 June event, the spacecraft resided in a higher-latitude part of the plasma sheet before the crossing, as is inferred from the fact that both the magnitude and the $x$ component of the magnetic field were more intense and the temperature was lower than in the 30 June event. The spacecraft traversals of the magnetopause took place in the time order $\mathrm{C} 4, \mathrm{C} 1, \mathrm{C} 2$, and $\mathrm{C} 3$, i.e. opposite to the order in the previous event. We see that the duration of the current layer traversal was relatively short for $\mathrm{C} 4$ and $\mathrm{C} 1$, whereas it was longer for $\mathrm{C} 2$ and $\mathrm{C} 3$. The local magnetosheath magnetic field was tailward/dawnward/southward, as in the previous event.

\subsection{Reconstruction from spacecraft 1 crossing}

The MVABC and HT analysis for the interval 06:23:0306:23:44 UT yield (all vectors are in GSE): the magnetopause normal vector, $\boldsymbol{n}=(0.6098,-0.7862,0.0999)$; the constant HT frame velocity, $V_{H T}=(-248.6,-102.5$, $68.6) \mathrm{km} / \mathrm{s}$ with the correlation coefficient, $c c_{H T}=0.9660$ (These two vectors are very close to, but not identical to those reported in Haaland et al. (2004)). The usage of the constraint $\left\langle B_{n}\right\rangle=0$ might be questionable for this event, since, as shown later, the Walén relation is relatively well satisfied, i.e. the boundary may be of the rotational discontinuity-type. Nonetheless, we use the constraint because the orientation of the normal with, rather than without, the constraint is more consistent with those from various other methods (Haaland et al., 2004). Also the result without the constraint leads to an unlikely large $B_{n}$ value. The normal component of the HT velocity is negative $\left(\boldsymbol{V}_{H T} \cdot \boldsymbol{n}=-64.1 \mathrm{~km} / \mathrm{s}\right)$, consistent with an outbound crossing of the magnetopause. The field map recovered from the $\mathrm{C} 1$ measurements during the time interval of 06:22:11 to 06:24:20 UT is shown in Fig. 9. For this event, the constant HT velocity is used for the reconstruction, because effects other than the kinematic effects of HT frame acceleration could be substantial, as will be shown later in this section. The optimal invariant axis is found to be $z=(0.6066,0.3061,-0.7337)(\mathrm{GSE})$ and $\mathrm{C} 2$, $\mathrm{C} 3$, and C4 were displaced from C1 by $-1219 \mathrm{~km},+935 \mathrm{~km}$, and $-570 \mathrm{~km}$, respectively, in the $z$ direction. In the reconstruction plane, the magnetosphere $\left(B_{x}>0 ; B_{y}<0\right)$ is on the lower left side and the magnetosheath $\left(B_{x}<0 ; B_{y}>0\right)$ on the upper right side. The magnetopause appears to be a slightly

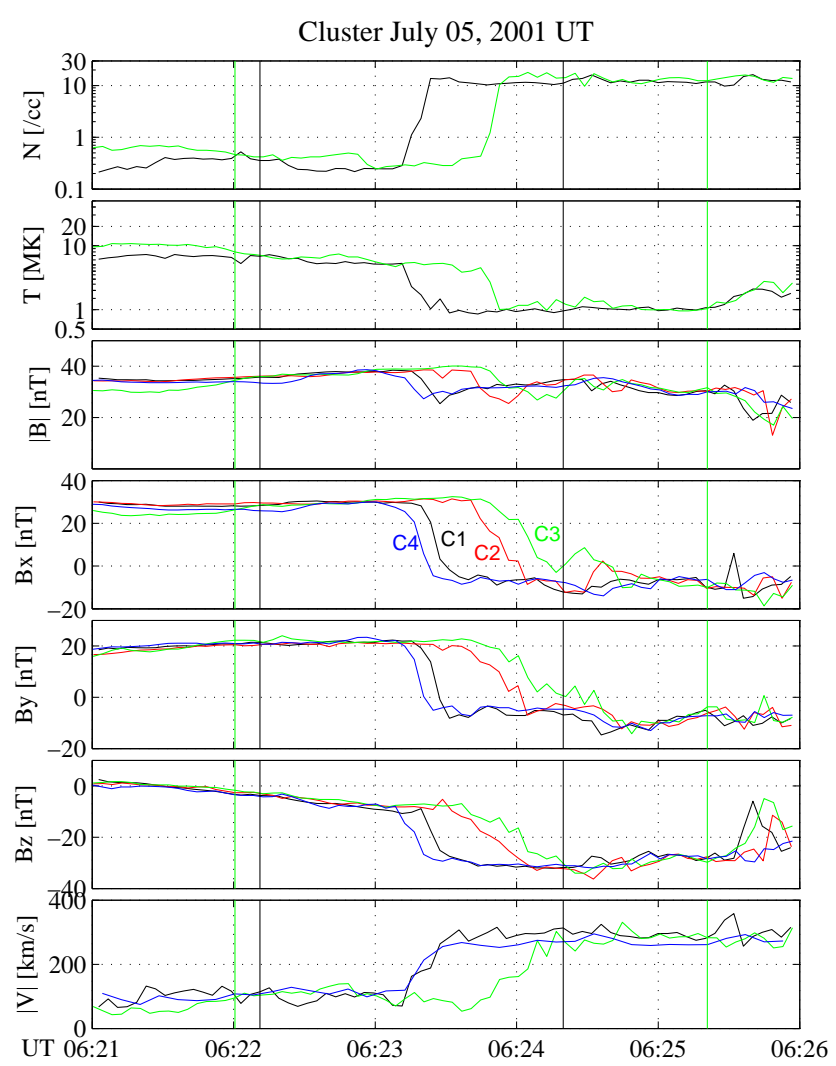

Fig. 8. Time plots of Cluster measurements around a magnetopause crossing event occurring at $(-6.78,-14.97,6.24) R_{E}$ in GSE on 5 July 2001. The format is the same as in Fig. 1.

bent TD-like structure. Thinning of the current sheet locally at $(x, y) \approx(9000,1000) \mathrm{km}$ implies the presence of an $X$ point at this location. The flow velocities remaining in the HT frame are shown by the white arrows. They are negligibly small on the magnetosheath side, indicating that, as before, the HT frame is strongly anchored in the magnetosheath plasma. Near the magnetopause on its magnetospheric side, the flow directions in the $\mathrm{C} 1$ and $\mathrm{C} 3$ crossings are consistent with the recovered field configuration. The yellow arrows, representing the normal vectors determined from MVABC for each spacecraft measurement are approximately perpendicular to the recovered magnetopause surface.

Figure 10 shows the result of the Walén test across the $\mathrm{C} 1$ magnetopause crossing, in which GSE velocity components in the HT frame are plotted against the corresponding components of the Alfvén velocity. The regression line has a significant positive slope (slope $=0.568$ ), suggesting that some reconnection activity could have been present. The positive slope means that the plasma was flowing parallel to the field, which has a small negative normal component, $B_{n}$, at the location of $\mathrm{C} 1$. In other words, one may infer that plasma was flowing earthward across the magnetopause, albeit at considerably less than Alfvénic speeds. These results are consistent with the reconstructed field map, although the $\mathrm{C} 1$ velocity vectors shown in the map do not show clear direct evidence for such an earthward flow component. 


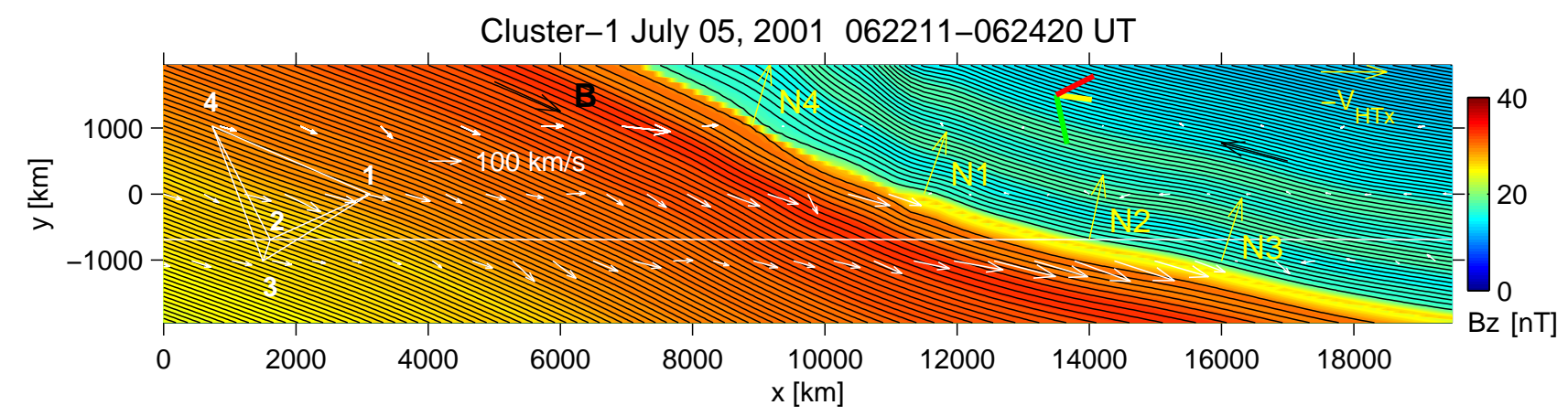

Fig. 9. Magnetic field map reconstructed by using a constant HT velocity for the C1 magnetopause crossing on 5 July 2001 . The format is the same as in the upper panel of Fig. 3, except that for $\mathrm{C} 1, \mathrm{C} 3$, and $\mathrm{C} 4$, the flow vectors in the HT frame are projected as white arrows, and for $\mathrm{C} 2$, the spacecraft trajectory is shown by a white curve. In this plane the magnetotail $\left(B_{x}>0 ; B_{y}<0\right)$ is on the lower left side, whereas the magnetosheath $\left(B_{x}<0 ; B_{y}>0\right)$ is on the upper right side. The yellow arrows show the projections of the normal vectors determined from MVABC.

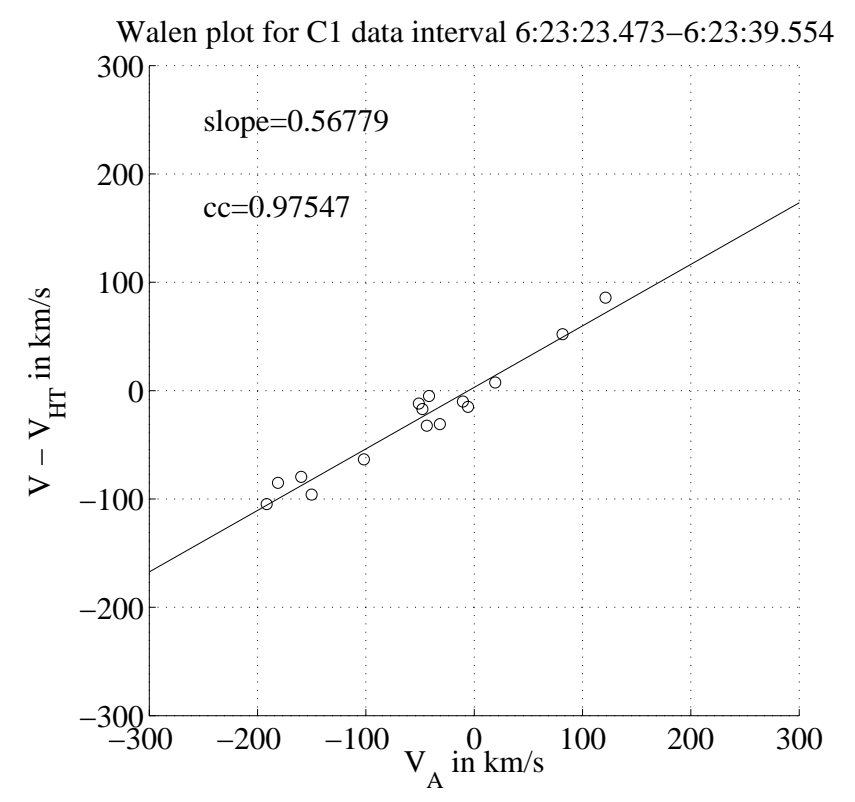

Fig. 10. Walén relation across the magnetopause encountered by $\mathrm{C} 1$ at $06: 23 \mathrm{UT}$ on 5 July 2001 . $\boldsymbol{V}_{\boldsymbol{H} T}=(-242.65,-84.71$, $162.28) \mathrm{km} / \mathrm{s}$ in GSE.

In Fig. 11 the correlation between the field components measured by $\mathrm{C} 2, \mathrm{C} 3$, and $\mathrm{C} 4$ and the corresponding components predicted from the reconstruction map (Fig. 9) are shown. The correlation is slightly lower than in the previous event but it remains high, demonstrating that the reconstruction technique works well also for this case. A few outlying points from the $B_{x}$ component of the $\mathrm{C} 4$ data result from a small error in the predicted time of the crossing by $\mathrm{C} 4$.

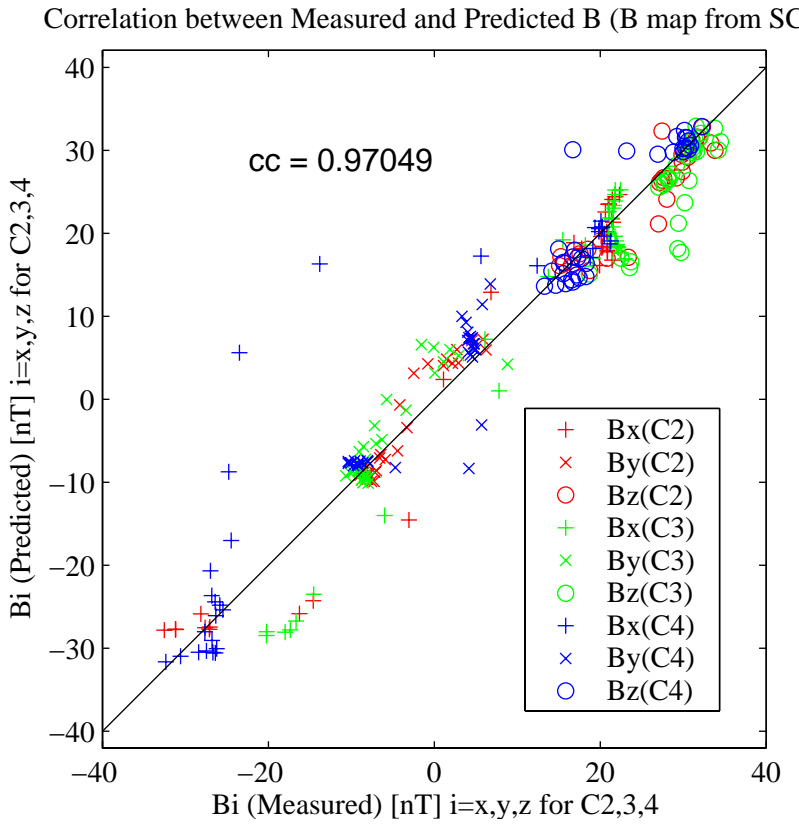

Fig. 11. Correlation between the measured and predicted magnetic field data. The predicted data are from the field map recovered for the C1 traversal on 5 July 2001 (Fig. 9).

\subsection{Reconstruction from spacecraft 3 crossing}

The MVABC and HT analysis for the interval 06:23:3206:24:49 UT yield: $\boldsymbol{n}=(0.5959, \quad-0.8000, \quad 0.0704)$; the constant HT frame velocity, $\boldsymbol{V}_{H T}=(-236.0,-94.5$, $125.4) \mathrm{km} / \mathrm{s}$ with the correlation coefficient, $c c_{H T}=0.9512$; and $\boldsymbol{V}_{H T} \cdot \boldsymbol{n}=-56.2 \mathrm{~km} / \mathrm{s}$. The GSE $z$ component of the HT velocity is substantially different from that computed for the $\mathrm{C} 1$ traversal; a possible explanation will be mentioned later. The normal motion of the magnetopause is negative, i.e. earthward, as required, although Haaland et al. (2004) have shown from Minimum Faraday Residue (MFR) analysis that the inward magnetopause speed was only some $43 \mathrm{~km} / \mathrm{s}$. 


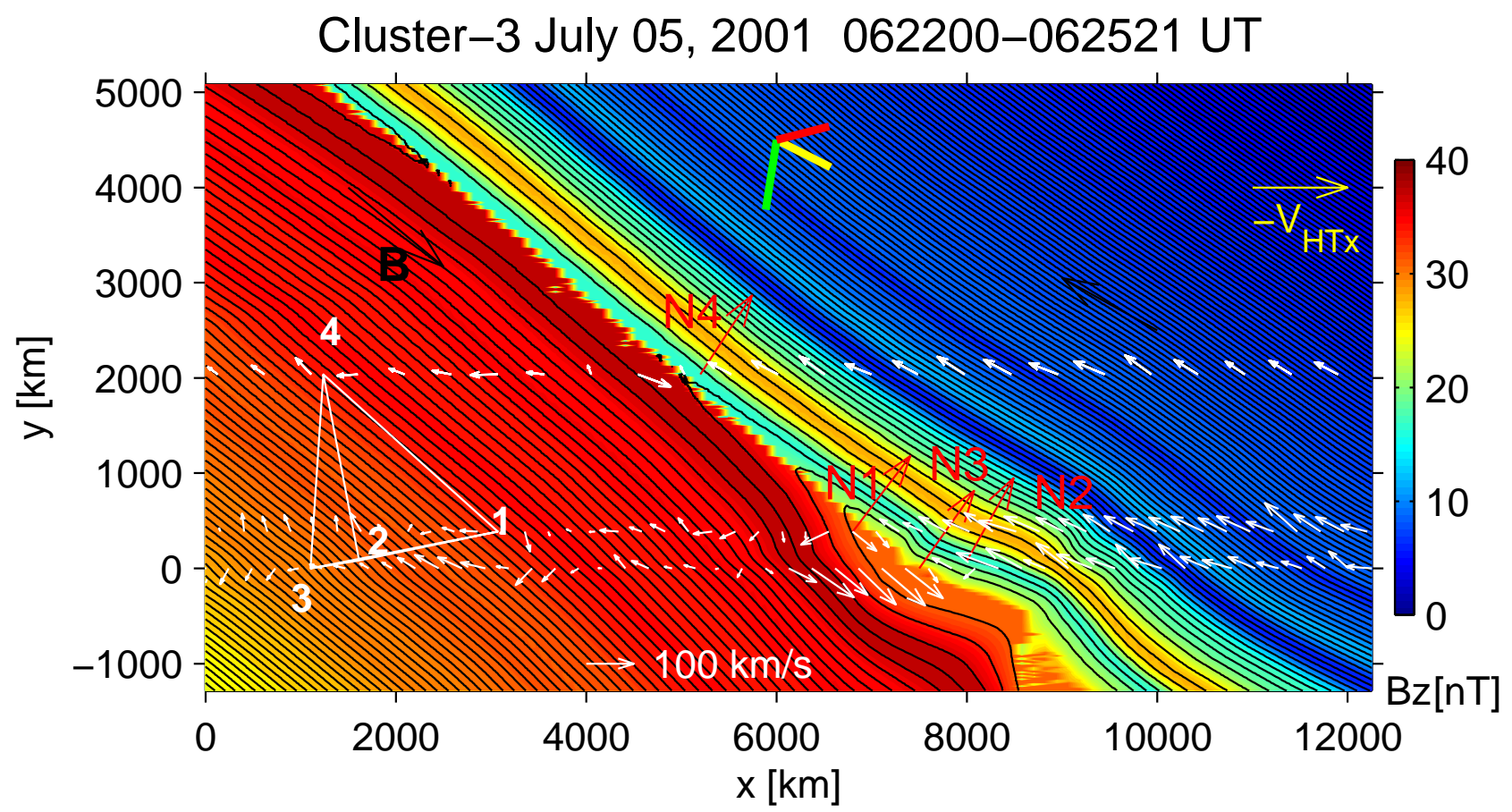

Fig. 12. Magnetic field map reconstructed for the C 3 magnetopause crossing on 5 July 2001. The format is the same as in Fig. 9 , except that the normal vectors are shown as red arrows.

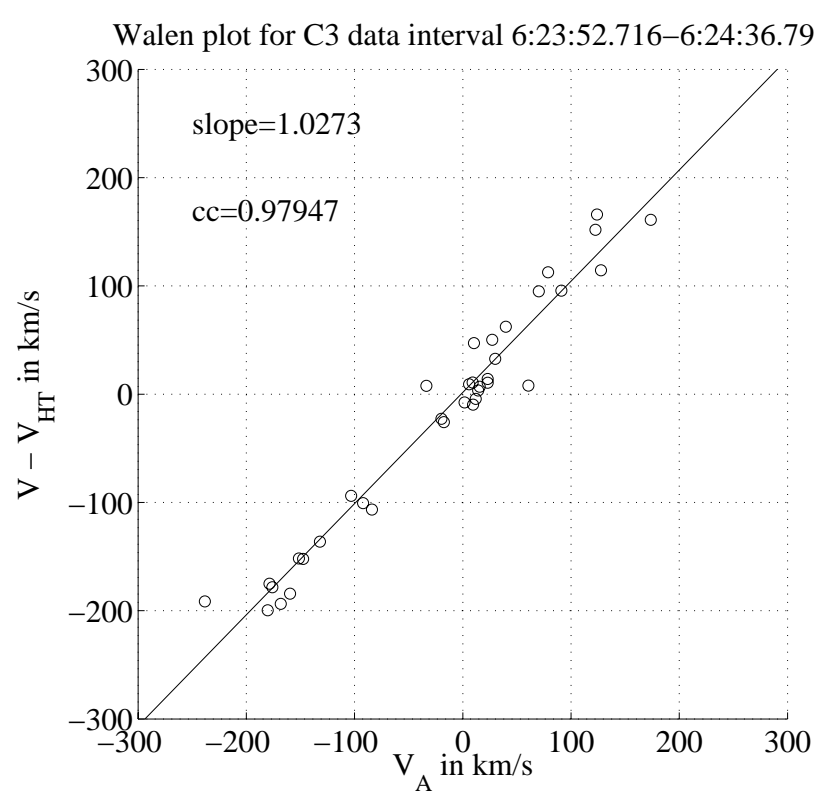

Fig. 13. Walén relation for the $\mathrm{C} 3$ magnetopause crossing occurring at 06:24 UT on 5 July 2001. $\boldsymbol{V}_{\boldsymbol{H} T}=(-254.11,-95.92$, $225.78) \mathrm{km} / \mathrm{s}$ in GSE. The Walén slope close to +1 is consistent with a rotational discontinuity magnetopause, with the magnetic field having an inward component.

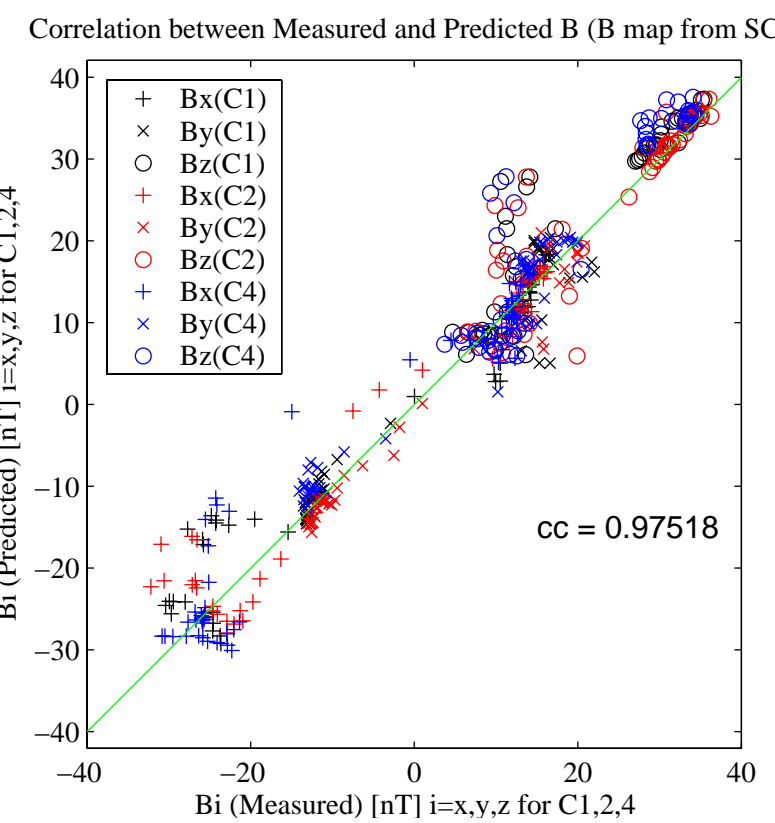

Fig. 14. Correlation between measured and predicted magnetic field data. The predicted data are based on the map reconstructed from the C3 data for the 5 July 2001 event (Fig. 12). 

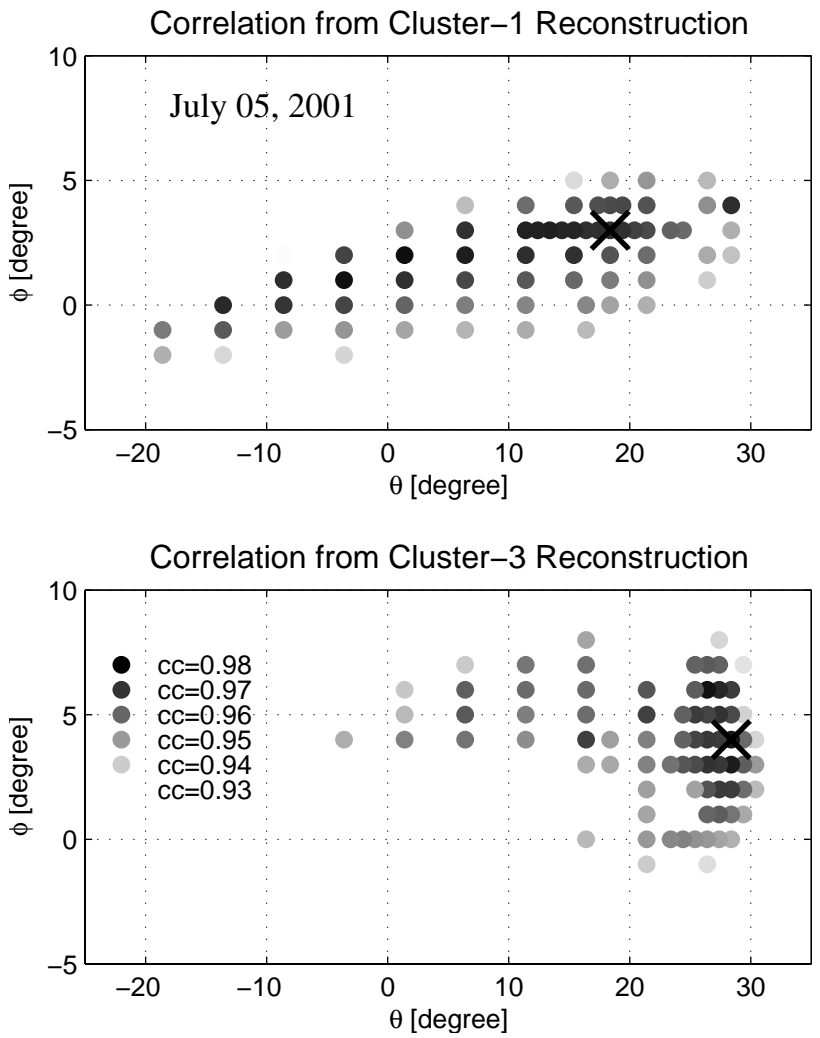

Fig. 15. Dependence of the correlation coefficient on the choice of the invariant axis for the reconstructions from the $\mathrm{C} 1$ (upper panel) and C3 (lower) traversals on 5 July 2001. The format is the same as in Fig. 7.

The difference between this number and $56.2 \mathrm{~km} / \mathrm{s}$ indicates the presence of an inward flow of plasma across the magnetopause. The magnetic field map reconstructed for this C3 magnetopause crossing, using the data from 06:22:00 to 06:25:21 UT, is shown in Fig. 12. The selected optimal invariant axis is $z=(0.6997,0.3727,-0.6096)$ (GSE), which is tilted from the invariant axis used in Fig. 9 by $9.7^{\circ}$. A significant amount of field lines that connect the magnetosheath and magnetospheric sides of the magnetopause is seen in the map. A prominent $X$ point in the transverse field at $(x, y) \approx$ $(4000,3000) \mathrm{km}$ looks more like a $Y$ point. The HT frame is no longer anchored in the magnetosheath plasma, i.e. the flow vectors have substantial field-aligned components in the magnetosheath. This behavior is suggestive of ongoing reconnection. Note that the plasma is flowing across the magnetopause in the direction parallel to the magnetic field in the open-field channel between the $X$ point and the center of a bulge in the current layer, located at $(x, y) \approx(7500,0) \mathrm{km}$, indicating that the magnetosheath plasma enters the magnetosphere along the reconnected field lines. The flow vectors have significant downward and rightward components at the bulge center, implying that in the reconstruction frame the reconnected flux tubes were moving in this direction. Notice that the spatial dimension of the map in the $x$ direction is smaller than in Fig. 9, in spite of the longer data analysis interval (see Fig. 8). This is due to a smaller HT frame speed along the $x$-axis for the $\mathrm{C} 3$ traversal, caused by the frame motion being better anchored in the reconnected field lines than for the $\mathrm{C} 1$ traversal.

The Walén plot for the $\mathrm{C} 3$ crossing is shown in Fig. 13. The flow speed in the HT frame is almost $100 \%$ of the Alfvén speed, in excellent agreement with the expectation from a one-dimensional RD. For earthward plasma flow across the magnetopause, the positive slope of the regression line implies that the normal magnetic field also points inward. This is consistent with the field map and with reconnection occurring tailward of the spacecraft. As in the 30 June event, the reconnection site is moving relative to Earth with a tailward velocity component.

Comparison of the two magnetic field maps for this event (Figs. 9 and 12) shows that there was dramatic evolution of the configuration during the 30-s time interval between the traversals by $\mathrm{C} 1$ and $\mathrm{C} 3$. At the moment when $\mathrm{C} 1$ crossed the current layer, there was incipient reconnection, as suggested by the corresponding Walén plot (Fig. 10). On the other hand, it is clear that when $\mathrm{C} 3$ crossed the magnetopause, the reconnection was fully developed and had resulted in the formation of a wide channel of interconnected field lines. The full-blown reconnection caused a localized thickening of the magnetopause current layer in the region traversed by $\mathrm{C} 3$ and an associated longer duration of this crossing (see Fig. 8). The crossing by $\mathrm{C} 2$ also had a long duration, which may, however, have been the result, at least in part, of a smaller magnetopause speed (Haaland et al., 2004). Such changes in speed are not accommodated by the map, which is based on a constant HT velocity.

Figure 14 shows the same type of correlation plot as Fig. 11, except that the predicted values are based on the map shown in Fig. 12. The correlation $(c c=0.975)$ suggests that the technique predicts conditions at the other three spacecraft locations fairly well. This result may be surprising since the map is derived under the assumption that inertia forces are small, which is not the case near the bulge where the streamlines have strong curvature and where the flow speed in the HT frame is comparable to the Alfvén speed (Fig. 13). The assumption that the time dependence of the structures is negligible is also not valid for this event, as is evident from a comparison of the maps in Figs. 9 and 12. Nevertheless, our results indicate that the maps recovered from $\mathrm{C} 1$ and $\mathrm{C} 3$ are at least qualitatively correct.

\subsection{Orientation of invariant axis}

In Fig. 15 the dependence of the correlation between the measured and predicted field components on the choice of the invariant (z)-axis for the 5 July 2001, event is shown. In these coordinates, the intermediate variance direction from MVABC is oriented at $(\theta, \phi)=(0,0)$ for the $\mathrm{C} 1$ crossing, while it is at $(\theta, \phi) \approx(2,-1)$ for the $\mathrm{C} 3$ crossing. We also see in this event that the optimal invariant axis is not far from the intermediate variance direction for both reconstructions. 
As in the previous event, the reconstruction from the $\mathrm{C} 1$ data produces a correlation coefficient that depends strongly on $\phi$ but only weakly on $\theta$. This behavior may be understood in the following way. The three reconstructions in Figs. 3, 5, and 9 exhibit a magnetopause current layer that is modestly tilted with respect to the $x$-axis in the reconstruction plane. Hence, rotations around the minimum variance axis (changes in $\theta$ ) change the $B_{y}$ component in the reconstruction plane only weakly, and do not have a significant influence on average profiles of $A$ calculated along the spacecraft trajectory. It follows that the behavior of the function $P_{t}(A)$ and hence, the reconstruction result, have only a modest dependence on $\theta$. On the other hand, rotations around the maximum variance axis, i.e. changes in $\phi$, cause significant changes in $B_{y}$ and therefore, a strong dependence on $\phi$.

In contrast, we find the correlation coefficient to be sensitive to variations in both $\theta$ and $\phi$ for the $\mathrm{C} 3$ reconstruction on 5 July (see Fig. 12). This behavior may be related to features that were not seen for the other three cases: The magnetopause crossed by $\mathrm{C} 3$ was tilted more steeply, relative to the $x$-axis, it was of the RD-type, and it had a fairly large-scale 2-D structure, namely the reconnection-associated bulge. A study of more cases is required to determine which of these factors affect the sensitivity of the correlation to variations of the orientation of the $z$-axis.

\subsection{Summary of 5 July 2001 event}

Substantial differences in the two recovered maps indicate that the magnetic field configuration evolved dramatically in the $\approx 30$-s interval between the magnetopause crossings by $\mathrm{C} 1$ and $\mathrm{C} 3$. At the time of the crossing by $\mathrm{C} 1$, the magnetopause was basically a TD-type current layer but with a small amount of interconnected field lines embedded. The boundary crossed by $\mathrm{C} 3 \mathrm{had}$ a much thicker current layer of RD-type. The presence of a single dominant $X$ point and an associated reconnection layer is evident in the field map recovered for the $\mathrm{C} 3$ crossing, indicating that reconnection had been developing locally in a time period less than $30 \mathrm{~s}$. Although the model assumptions of time invariance and of negligible inertia forces are violated in the event, the bulge in the magnetopause, containing reconnected field lines in the C3 map, was found to be a persistent feature in our various reconstruction attempts. For this reason, we believe the C3 map to be at least qualitatively correct.

\section{Summary and discussion}

In this paper, we have applied the technique for recovering 2D magnetohydrostatic structures from single-spacecraft data to two magnetopause crossings by the four Cluster spacecraft, occurring when they were separated by about two thousand $\mathrm{km}$ from each other. In summary, the following results have been obtained.

1. An optimal invariant ( $z$ )-axis can be found in such a way that the correlation between the magnetic field components predicted from the reconstruction map for one spacecraft and the corresponding components measured by the other three is at, or near, a maximum, with the proviso that the measured velocity vectors, transformed into the co-moving (HT) frame, become nearly field-aligned in the field map (see Appendix A). The orientation of the invariant axis thus selected is relatively close to the intermediate variance direction determined by MVABC. The invariant axis is generally well determined with respect to rotations around the maximum variance axis but less well with respect to rotations around the minimum variance axis.

2. Two complete magnetopause crossings, occurring on 30 June 2001 and 5 July 2001, have been examined. For each of the two events, two reconstruction maps have been produced, one based on the data from $\mathrm{C} 1$ and a second based on the data from C3. For an optimally selected invariant (z)-axis and HT frame velocity, the correlation coefficient between the predicted and measured field components exceeds 0.97 in all four cases. The result demonstrates that the reconstruction technique is capable of predicting field behavior at distances up to a few thousand km away from the spacecraft used for the reconstruction.

3. The reconstruction method incorporating the slidingwindow HT analysis that takes into account time-varying motions of the HT frame, as described by Hu and Sonnerup (2003), was successfully applied to the 30 June 2001 event. This result suggests that, over a spatial scale of a few thousand $\mathrm{km}$, the entire portion of the magnetopause shown in a map was approximately time-stationary but was moving in a time-dependent way. Localized motions of the magnetopause were small.

4. Intercomparison of the two field maps obtained for the 30 June 2001 event shows that the overall magnetopause structures were similar in the two maps, having a current layer of TD-type. It appears that the assumptions of local two dimensionality and time coherence were well satisfied for the magnetopause encountered on this day. The reconstructed field structures show a current layer significantly bent on spatial scales of a few thousand $\mathrm{km}$, demonstrating that the magnetopause cannot always be treated as a planar structure during a Cluster encounter. Haaland et al. (2004) have shown that even modest deviations from the planar geometry can lead to difficulties with various multi-spacecraft techniques for predicting the magnetopause velocity.

5. In the 5 July 2001, event, time evolution is clear from comparison of two field maps recovered individually from $\mathrm{C} 1$ and $\mathrm{C} 3$, which crossed the magnetopause at different moments. Evidence consistent with reconnection developing locally in the magnetopause current layer over a time interval of $30 \mathrm{~s}$ or less has been found. The map recovered for $\mathrm{C} 3$ shows a rather thick current layer with a dominant $X$ point and interconnected flux tubes embedded, allowing for an efficient access of the magnetosheath plasma into the magnetosphere, while the map for $\mathrm{C} 1$, which spacecraft crossed the boundary $\sim 30 \mathrm{~s}$ earlier than $\mathrm{C} 3 \mathrm{did}$, shows a thin TD-type current sheet within which a much smaller amount of interconnected field lines is present. 
6. Density ramps at the magnetopause occurred in the earthmost half of the current layer in both events (see Figs. 1 and 8). This behavior is consistent with the recovered field maps which show a dominant $X$ point and associated flux tubes that connect the outer and inner parts of the magnetopause transition layer. The interconnection permits effective transport of magnetosheath plasma into most of the current layer, via field-aligned flow. The ramps were located in the inner half of the current sheet, for the $\mathrm{C} 1$ traversal in the 30 June 2001 event and for the $\mathrm{C} 3$ traversal in the 5 July 2001 event, whereas they were closer to the center of the current sheet, for the C 3 traversal in the June 30 event, and for the $\mathrm{C} 1$ traversal in the 5 July event. This can be explained by the temporal evolutions seen in the maps: for both events, the layer consisting of the interconnected field lines had been thickened during the interval between the $\mathrm{C} 1$ and $\mathrm{C} 3$ traversals. In neither event is there any evidence of a low-latitude boundary layer, containing magnetosheath-like plasma, earthward of, but adjoining, the magnetopause.

7. Our experiments have shown that the optimal invariant (z)-axis is not far away from the intermediate variance direction for the cases examined, but also that a modest rotation of the trial $z$-axis around the maximum variance direction is critical for optimization of the map. This could be related to the fact that the ratio of intermediate to minimum eigenvalues is often not very large, resulting in significant uncertainties in the determination of both minimum and intermediate variance directions (Sonnerup and Scheible, 1998). Proximity of the invariant axis to the intermediate variance direction suggests that MVABC can provide a rough estimation of the orientation of the axis of two-dimensional structures and hence, of $X$ lines, etc.

8. The orientation of the optimal invariant axis is not very different for the two events, which were at positions nottoo-distant from one another: The angle between the invariant axes for the $\mathrm{C} 1$ crossings is $\approx 25^{\circ}$. It is also noted that the orientation of the magnetic field outside of the magnetopause was relatively similar among the two events: The angle between the magnetosheath field directions for the 30 June 2001 and 5 July 2001 events is $15^{\circ}$. This result suggests that, at a chosen location on the magnetopause surface, the orientation of the reconnection lines is similar for similar IMF directions. This topic and also the question of how the orientation of the $X$ lines depends on the solar wind conditions are important subjects to be pursued in future work by applying the reconstruction method to more events.

9. Both events occurred on the tail flank magnetopause, on the dawn side. The signatures of the RD-type current layer, found for the 5 July 2001 event in both the reconstruction result and the Walén test for C3, suggest that reconnection can occur at the dawn tail magnetopause, consistent with the conclusion reached by Phan et al. (2001). But the local magnetic shear for the 5 July 2001 , event was not very high $\left(101^{\circ}\right)$, in contrast with the reconnection events reported by Gosling et al. (1986) and Phan et al. (2001). Those events also occurred at the tail flank magnetopause but under almost antiparallel field conditions. The present event is consistent with the finding that occurrence of reconnection on the tail surface is not rare even for relatively modest magnetic shears (Hirahara et al., 1997; Hasegawa, 2002; Hasegawa et al., 2004). It is noted in Fig. 12 that a clear $X$ point and significant out-of-plane magnetic field components are found within the reconstructed domain, demonstrating that component merging was occurring. Our results for the 5 July 2001 event also indicate that the reconnection site was not stationary relative to Earth but was moving both downstream and toward higher latitudes.

10. Although a qualitatively consistent field map was obtained for the C 3 crossing on 5 July 2001, the fact that the Walén slope was close to one (Fig. 13) indicates that inertia forces must have played an important role in the tangential stress balance in the reconnection layer. Incorporation of inertia effects into the reconstruction technique is not simple but is necessary for accurate modeling of magnetopause structures during significant reconnection activity, as on 5 July 2001. If such effects could be accurately taken into account, the recovered field map might show significant quantitative deviations from the one shown in Fig. 12, at least near the reconnection site. Even so, we expect the map in Fig. 12 to be qualitatively correct. Development and testing of a technique that incorporates inertia effects will be addressed in a future study.

11. The present work has made it clear that the past onedimensional (1-D) local view of the magnetopause is not adequate. The constrained normal, $\boldsymbol{n}$, from MVABC appears to represent the average magnetopause orientation relatively well, but the reconstructed maps show that the local orientations can deviate from the average. Mesoscale 2-D structures seen in the magnetopause current layer and their dependence on parameters on the two sides will provide insights into how reconnection operates and how the mesoscale phenomena are controlled by the plasma parameter regime. These problems will be dealt with in a future statistical study.

\section{Appendix A Optimizing the invariant ( $z$ )-axis, HT frame, and extrapolations of $P_{t}(A)$}

In this Appendix, we describe the steps taken to find an optimal invariant axis, HT frame, and extrapolations of the transverse pressure function $P_{t}(A)$. In the present study, we try to determine the above parameters basically in such a way that the correlation between the measured and predicted magnetic field components becomes higher. This process is justified since, under the model assumptions, variations in time series data measured by the spacecraft should translate directly into spatial variations along the trajectory of spacecraft across static magnetic field structures, i.e. they should be caused by motion of the structures past the spacecraft. We use the reconstruction from $\mathrm{C} 1$ on 30 June 2001, at 18:12 UT as a vehicle for the presentation but the steps described are general ones.

1. Initially, the HT frame, i.e. the motion of the local structures past the spacecraft, which is required for determination 
of the spatial scale of the reconstruction domain in the $x$ direction and for computation of the magnetic vector potential, $A$, is determined. Under the assumption of time independence of the structures, acceleration or/and rotation of the HT frame is allowed, but as a first step we simply use a constant HT frame velocity obtained from $\mathrm{C} 1$ for the interval 18:12:00-18:12:49 UT.

2. We define $L, M$, and $N$ axes as the maximum, intermediate, and minimum variance directions, respectively, which are determined from MVABC (Appendix B) and are ordered as a right-handed orthogonal coordinate system with $N$ pointing outward. An optimal invariant axis is searched for by rotating the trial $z$-axis by trial and error, starting from the intermediate variance $(M)$ direction, such that the correlation coefficient in Fig. 4b, between the measured and predicted field components, reaches a higher value. First the initial invariant axis (the $M$ axis) is rotated in the plane perpendicular to $N$ by an angle $\theta$ from the $M$ direction. New axes $M^{\prime}$ and $L^{\prime}$ are determined after this rotation. The trial invariant $\left(M^{\prime}\right)$ axis thus obtained is then rotated by $\phi$ in the plane perpendicular to $L^{\prime}$, resulting in a new invariant axis $M^{\prime \prime}$, which is used for a trial reconstruction. The positive signs of $\theta$ and $\phi$ are defined according to the right-hand rule. A certain number of candidate orientations, $M^{\prime \prime}$, for which the correlation coefficient is sufficiently high, are chosen by surveying the two angles, $\theta$ and $\phi$. In principle, any coordinate system may be used for this survey process. In this study, we use the coordinate system based on the results from MVABC.

3. As shown in Fig. 7, the angular domain in which the correlation coefficient exceeds a certain value is belt-like and there is an uncertainty in the determination of an optimal $\theta$ value. Therefore, two further criteria are used to select the best invariant axis from the candidate orientations, one based on the functional behavior of $P_{t}(A), p(A)$, and $B_{z}(A)$, the other based on the alignment between the remaining velocity vectors in the HT frame and magnetic field lines, when visually inspected in the recovered map. For some of the candidates, the quantities $P_{t}, p$, or $B_{z}$ have two or more significantly different values for certain $A$ values near the center of the current sheet, i.e. near the maximum of $P_{t}$ and $A$ in the $P_{t}$ versus $A$ plot (see Fig. 2), meaning that they vary substantially on the same field line and thus, that the model assumptions are violated. For other cases, the velocity vectors in the HT frame of $\mathrm{C} 1$, measured by the spacecraft not used for the reconstruction ( $\mathrm{C} 3$ and $\mathrm{C} 4$ ), have non negligible components in the direction perpendicular to the reconstructed magnetic field lines. This feature suggests that the recovered field may not be reasonable. The best orientation of the invariance $(z)$ is determined by considering these features.

4. In a second cycle of trial and error, the reconstruction is tested by incorporating the sliding-window HT technique (Hu and Sonnerup, 2003), which allows for the acceleration of the HT frame. This step is taken unless a very nearly timeindependent HT frame is found, that is unless the correlation coefficient between components of $-\boldsymbol{V} \times \boldsymbol{B}$ and $-\boldsymbol{V}_{H T} \times \boldsymbol{B}$ for the analysis interval is extremely good. Note that the ap- plication of this method assumes that the entire structure encountered by the four spacecraft moves together with a timevarying HT velocity. Inertia effects associated with the acceleration of the HT frame are assumed to be negligible. The optimal invariant axis can then be selected in the same way as in steps 2 and 3. If a better correlation is obtained than for the constant HT velocity case, the result obtained by using the time-varying HT velocity is adopted as the optimal one. Otherwise, the result with the constant HT velocity is selected.

5. If a less than satisfactory correlation is obtained for both the constant and the time-varying HT velocity cases, a modified method to compute the HT velocity that results in larger acceleration of the HT frame, described in Sect. 2.3, can be tested to improve the result.

6. Our experience indicates that the correlation coefficient depends relatively strongly on the choice of both the invariant axis and the HT frame velocity, but only weakly on the behavior of the extrapolating exponential functions in the $P_{t}$ versus $A$ plot. Therefore, these exponential functions are adjusted after the above steps are finished. The above behavior is reasonable, since the extrapolating functions only modify magnetic field values in regions far from the current sheet, but have no effect on the shape of the current sheet. The correlation coefficient seems most sensitive to how well the timing of the magnetopause crossings is predicted.

\section{Appendix B Intermediate and maximum variance axes with the constraint $\left\langle B_{n}\right\rangle=0$}

Methods for determining the vector normal to the magnetopause with the constraint $\left\langle B_{n}\right\rangle=0$ were given by Sonnerup and Scheible (1998). Here we describe a method to determine the intermediate and maximum variance directions under this constraint.

By using a constraint of the form $\hat{\boldsymbol{n}} \cdot \hat{\boldsymbol{e}}=0$, where $\hat{\boldsymbol{e}}$ is a known unit vector (here to be chosen as the normal vector from MVABC, $\boldsymbol{n}_{\left\langle B_{n}\right\rangle=0}$, the eigenvalue problem can be written,

$\mathbf{P} \cdot \mathbf{M}^{B} \cdot \mathbf{P} \cdot \hat{\boldsymbol{n}}=\lambda \hat{\boldsymbol{n}}$.

Here, $\mathbf{M}^{B}$ is the magnetic variance matrix, $\mathbf{M}_{i j}^{B} \equiv\left\langle B_{i} B_{j}\right\rangle-\left\langle B_{i}\right\rangle\left\langle B_{j}\right\rangle$, and $\mathbf{P}$ is the matrix describing the projection of a vector onto the plane perpendicular to $\hat{\boldsymbol{e}}$, i.e. $\mathbf{P}_{i j}=\delta_{i j}-e_{i} e_{j}$. By putting $\hat{\boldsymbol{n}}=\hat{\boldsymbol{e}}$ in the eigenvalue Eq. (B1), it is seen that $\hat{\boldsymbol{e}}$ is an eigenvector corresponding to $\lambda=0$. The other two eigenvalues are denoted by $\lambda_{\min }$ and $\lambda_{\max }$. The eigenvectors corresponding to $\lambda_{\min }$ and $\lambda_{\max }$ represent the minimum and maximum variance directions, respectively, in the plane perpendicular to $\hat{\boldsymbol{e}}$. Thus, for $\hat{\boldsymbol{e}}=\boldsymbol{n}_{\left\langle B_{n}\right\rangle=0}$, the eigenvectors for $\lambda_{\min }$ and $\lambda_{\max }$ represent the intermediate and maximum variance directions, respectively, under the constraint $\left\langle B_{n}\right\rangle=0$. If one puts $\hat{\boldsymbol{e}}=\frac{\langle\boldsymbol{B}\rangle}{|\langle\boldsymbol{B}\rangle|}$ instead, the eigenvector corresponding to $\lambda_{\min }$ is the normal vector from MVABC (Sonnerup and Scheible, 1998). 
Acknowledgements. H. H. thanks Qiang Hu for fruitful discussions and for his instruction in Matlab programming. Work at Dartmouth College was supported by NASA grant NAG 5-12005. The research was initiated with H. H. and BUÖS in residence at the Max-PlanckInstitut für extraterrestrische Physik, Garching.

Topical Editor T. Pulkkinen thanks two referees for their work in evaluating this paper.

\section{References}

Balogh, A., Carr, C. M., Acuna, M. H., et al.: The Cluster magnetic field investigation: Overview of in-flight performance and initial results, Ann. Geophys., 19, 1207-1217, 2001.

Elphic, R. C.: Observations of flux transfer events: A review, Physics of the magnetopause, Geophys. Monogr. Ser., vol. 90, edited by P. Song, B. U. Ö Sonnerup, and M. F. Thomsen, AGU, Washington, D. C., 225-233, 1995.

Gosling, J. T., Thomsen, M. F., Bame, S. J., and Russell, C. T.: Accelerated plasma flows at the near-tail magnetopause, J. Geophys. Res., 91, 3029-3041, 1986.

Haaland, S. E., Sonnerup, B. U. Ö., Dunlop, M. W., Balogh, A., Georgescu, E., Hasegawa, H., Klecker, B., Paschmann, G., PuhlQuinn, P., Rème, H., Vaith, H., and Vaivads, A.: Four-spacecraft determination of magnetopause orientation, motion, and thickness: Comparison with results from single-spacecraft methods, Ann. Geophys., in press, 2004.

Hasegawa, H.: Entry process of the solar wind plasma into the Earth's magnetotail, Ph. D. thesis, Nagoya Univ., Japan, 2002.

Hasegawa, H., Maezawa, K., Saito, Y., and Mukai, T.: Relationship between field-aligned electron fluxes and field line topology at the tail lobe magnetopause, Adv. Space Res., submitted, 2004.

Hau, L.-N. and Sonnerup, B. U. Ö.: Two-dimensional coherent structures in the magnetopause: Recovery of static equilibria from single-spacecraft data, J. Geophys. Res., 104, 6899-6917, 1999.

Hirahara, M., Terasawa, T., Mukai, T., Hoshino, M., Saito, Y., Machida, S., Yamamoto, T., and Kokubun, S.: Cold ion streams consisting of double proton populations and singly charged oxygen observed at the distant magnetopause by GEOTAIL: A case study, J. Geophys. Res., 102, 2359-2372, 1997.
Hu, Q. and Sonnerup, B. U. Ö.: Magnetopause transects from two spacecraft: A comparison, Geophys. Res. Lett., 27, 1443-1446, 2000.

$\mathrm{Hu}$, Q. and Sonnerup, B. U. Ö.: Reconstruction of magnetic flux ropes in the solar wind, Geophys. Res. Lett., 28, 467-470, 2001.

Hu, Q. and Sonnerup, B. U. Ö.: Reconstruction of magnetic clouds in the solar wind: Orientation and configuration, J. Geophys. Res., 107(A7), 1142, doi:10.1029/2001JA000293, 2002.

Hu, Q., and Sonnerup, B. U. Ö.: Reconstruction of two-dimensional structures in the magnetopause: Method improvements, J. Geophys. Res., 108(A1), 1011, doi:10.1029/2002JA009323, 2003.

Hu, Q., Smith, C. W., Ness, N. F., and Skoug, R. M.: Double fluxrope magnetic cloud in the solar wind at $1 \mathrm{AU}$, Geophys. Res. Lett., 30(7), 1385, doi:10.1029/2002GL016653, 2003.

Khrabrov, A. V., and Sonnerup, B. U. Ö.: DeHoffmann-Teller Analysis, in: Analysis Methods for Multi-Spacecraft Data, ISSI Sci. Rep., SR-001, 221, Kluwer Acad., Norwell, Mass., 221-248, 1998.

Phan, T.-D., Sonnerup, B. U. Ö., and Lin, R. P.: Fluid and kinetics signatures of reconnection at the dawn tail magnetopause: Wind observations, J. Geophys. Res., 106, 25 489-25 501, 2001.

Rème, H., Aoustin, C., Bosqued, J. M., et al.: First multispacecraft ion measurements in and near the Earth's magnetosphere with the identical Cluster ion spectrometry (CIS) experiment, Ann. Geophys., 19, 1303-1354, 2001.

Sonnerup, B. U. Ö. and Guo, M.: Magnetopause transects, Geophys. Res. Lett., 23, 3679-3682, 1996.

Sonnerup, B. U. Ö. and Scheible, M.: Minimum and maximum variance analysis, in Analysis Methods for Multi-Spacecraft Data, ISSI Sci. Rep., SR-001, 185, Kluwer Acad., Norwell, Mass., 185-220, 1998.

Sturrock, P.: Plasma Physics, An Introduction to the Theory of Astrophysical, Geophysical and Laboratory Plasmas, Cambridge Univ. Press, New York, 209, 1994. 